\title{
Changes in Organic and Inorganic Osmolytes of Maize (Zea mays L.) by Sulfur Application Under Salt Stress Conditions
}

\author{
Alia Riffat $^{1} \&$ Muhammad Sajid Aqeel Ahmad ${ }^{1}$ \\ ${ }^{1}$ Department of Botany, University of Agriculture, Faisalabad, Pakistan \\ Correspondence: Alia Riffat, University of Agriculture, Faisalabad, Pakistan. E-mail: aliariffat@hotmail.com
}

Received: August 9, 2018

Accepted: September 21, $2018 \quad$ Online Published: November 15, 2018

doi:10.5539/jas.v10n12p543

URL: https://doi.org/10.5539/jas.v10n12p543

\begin{abstract}
Mineral nutrients have favourable potential in alleviation of salinity problem in plants. Sulfur has specific functions in regulating plant growth, metabolism, enzymatic reactions and osmolyte homeostasis in plants. Hence, an experiment was carried out to explore the role of sulfur in ameliorating salt toxicity in maize by changes in organic and inorganic osmolyte contents. A range of sulfur levels $(40,80 \mathrm{mM})$ were used to induce salinity tolerance in maize. Various treatments of salinity $(25,75 \mathrm{mM})$ were applied by using sodium chloride. Results revealed that glycine betaine, proline, total soluble sugars, total soluble proteins and total free amino acids contents were increased by applying salinity while the application of sulfur lowered the proline and increased other studied organic osmolyte contents in all studied maize organs (leaf, shoot, root). The maximum improvement in organic osmolyte contents were found at $40 \mathrm{mM}$ sulfur, however, at $80 \mathrm{mM}$ sulfur proline contents were reduced. Applied salinity increased leaf tissue concentration of $\mathrm{Na}^{+}$and decreased that of $\mathrm{K}^{+}, \mathrm{Ca}^{2+}$, $\mathrm{NO}_{3}{ }^{-}, \mathrm{PO}_{4}{ }^{3-}, \mathrm{SO}_{4}{ }^{2-}$ leading to a severely declined in $\mathrm{K}^{+} / \mathrm{Na}$ and $\mathrm{Ca}^{2+} / \mathrm{Na}^{+}$ratio. However, application of sulfur reduced the $\mathrm{Na}^{+}$contents and improved $\mathrm{K}^{+}, \mathrm{Ca}^{2+}, \mathrm{NO}_{3}{ }^{-}, \mathrm{PO}_{4}{ }^{3-}, \mathrm{SO}_{4}{ }^{2-}, \mathrm{K}^{+} / \mathrm{Na}^{+}$and $\mathrm{Ca}^{2+} / \mathrm{Na}^{+}$ratio in the salinity grown plants. Moreover, $40 \mathrm{mM}$ level of sulfur was greatly effective in osmolyte homeostasis at all levels of salinity. This indicated that use of sulfur $(40 \mathrm{mM})$ ameliorated the effect of salinity by changing organic and inorganic osmolyte contents in maize plants.
\end{abstract}

Keywords: ions, maize, osmolytes, sulfur, salinity

\section{Introduction}

Among various abiotic stresses, salt stress has affected $20 \%$ of land used for cultivation and $33 \%$ of the irrigated land throughout the world (Machado \& Serralheiro, 2017). Overall, 10 million ha of the world land has been degraded due to salinity each year (Pimentel et al., 2004). Salt stress causes disturbances in physiological, biochemical, molecular processes in the plant (Nahar et al., 2016). As a result osmotic stress, imbalance in nutrient transport and accumulation of reactive oxygen species takes place (Iqbal et al., 2014; Puniran-Hartley et al., 2014). In such conditions, plants synthesize and accumulate various organic and inorganic osmolytes or osmoprotectants. These include proline, glycine betaine, glucose, isoleucine, mannitol and proteins (Parida \& Das, 2005) and various inorganic nutrients $\left(\mathrm{K}^{+}, \mathrm{Ca}^{2+}, \mathrm{NO}_{3}{ }^{-}, \mathrm{PO}_{4}{ }^{3-}, \mathrm{SO}_{4}{ }^{2-}\right)$. The functions of these osmolytes are, to balance the ionic transport across the plant cell, scavenge reactive oxygen species, regulate enzyme activity and prevent membrane disintegration (Nahar et al., 2016). However, such strategies are needed that balances the concentrations of various osmolytes for maintaining plant metabolism. As higher concentration of osmolytes become toxic for plant cell.

Sulfur plays a significant role in balancing the osmolyte contents in the plants. Sulfur is a basic constituent of many important compounds that maintain plant growth and development in stress conditions. These compounds include glutathione, vitamins, phytoharmones and various co-enzymes (Spadaro et al., 2010). Sulfur helps in coordination among different physiological and biochemical processes in the plants. Hence, Sulfur improves the cellular function by balancing the organic and inorganic osmolytes that develops salt tolerance in crop plants (Taiz \& Zeiger, 2006; Nazar et al., 2014; Riffat \& Ahmad, 2016).

After wheat and rice, maize is very important cereal crop in the world. It is also known the 'king of crops'. It contains many types of vitamins and nutrients. Due to its nutritional importance it has become a valuable food and feed crop in many countries of world. It is used for making bread, cake and porridge. Also it is an important constitute of livestock and poultry diet (Bukhsh et al., 2011). However, the production and quality of maize is 
seriously affected by salinity as maize is moderately sensitive to salinity (Farooq et al., 2015). Therefore, such methods should be devised that increase the salt tolerance of this valuable crop to meet the growing food demand.

Hence, this study focuses on the improvement in salt tolerance potential of maize by sulfur application. To maintain the balance of organic and inorganic osmolytes for development of salt tolerance is another objective of this study.

\section{Method}

\subsection{Plan of Study}

A study was conducted to determine the role of sulfur in enhancing salt tolerance by changing the osmolyte contents in maize. The seeds of maize cultivars (Agaitti, 2003; Pak Afgoi, 2003) were acquired from Maize and Millet Institute Sahiwal Pakistan. The seeds were sorted and 10 uniform seeds were sown in plastic pots filled with $10 \mathrm{~kg}$ soil.

\subsection{Treatment Application}

Salinity $(25,75 \mathrm{mM})$ was applied by using sodium chloride. Various levels of sulfur $(40,80 \mathrm{mM})$ were applied by using potassium sulfate. Both treatments were applied at sowing time. After 15 days of treatment application, sulfur $(40,80 \mathrm{mM})$ was applied as foliar spray. Then 45 days plants were harvested for the determination of various biochemical attributes.

\subsection{Determination of Organic Osmolytes}

\subsubsection{Glycine Betaine}

Grieve and Grattan (1983) proposed a procedure for the determination of glycine betaine contents. Two reagents $2 \mathrm{~N} \mathrm{H}_{2} \mathrm{SO}_{4}$ and IK- $\mathrm{I}_{2}$ were prepared. $2 \mathrm{~N} \mathrm{H}_{2} \mathrm{SO}_{4}$ was prepared by mixing $5.6 \mathrm{~mL}$ of $36 \mathrm{M} \mathrm{H}_{2} \mathrm{SO}_{4}$ and distilled water was used for making final volume $100 \mathrm{~mL}$. IK- $\mathrm{I}_{2}$ was made by mixing $20 \mathrm{~g}$ of potassium iodide, $100 \mathrm{~mL}$ water and $15.7 \mathrm{~g}$ of iodine. Glycine betaine contents were determined by grounding $0.5 \mathrm{~g}$ dried plant material in $20 \mathrm{~mL}$ of deionized water and shaken for $24 \mathrm{~h}$ at $25{ }^{\circ} \mathrm{C}$. The extract was filtered and diluted with $2 \mathrm{~N} \mathrm{H}_{2} \mathrm{SO}_{4}$ in 1:1 ratio. Then $0.5 \mathrm{~mL}$ extract was put in centrifuge tube and kept in ice cooled water for 1 hour followed by addition of $1 \mathrm{~mL}$ of $\mathrm{IK}_{2} \mathrm{I}_{2}$, and vortexed at $0{ }^{\circ} \mathrm{C}$ at $10,000 \mathrm{~g}$ for $15 \mathrm{~min}$. The supernatant was collected and dissolved in $9 \mathrm{~mL}$ of 1-2 dichloroethane. The solution was kept at room temperature for 2-2.5 h. The absorbance of glycine betaine was noted at $365 \mathrm{~nm}$ by using spectrophotometer (UV-1100). The values were compared with standard curve.

\subsubsection{Proline}

Proline contents in plants were determined by the procedure proposed by Bates et al. (1973). Firstly, some reagents were prepared. $6 \mathrm{M}$ phosphoric acid was prepared by diluting $407 \mathrm{~mL}$ of $85 \%$ phosphoric acid in 1000 $\mathrm{mL}$ distilled water. For the preparation of acid-ninhydrin, $1.25 \mathrm{~g}$ of ninhydrin was dissolved in $30 \mathrm{~mL}$ glacial acetic acid and $20 \mathrm{~mL}$ of $6 \mathrm{M}$ phosphoric acid. 3\% sulfuric acid was made by mixing $3 \mathrm{~g}$ of sulphosalicylic acid in $100 \mathrm{~mL}$ of distilled water. For the determination of proline contents in plant material $0.1 \mathrm{~g}$ fresh plant sample was homogenised in $10 \mathrm{~mL}$ of $3 \%$ sulphosalicylic acid and filtered. Then $2 \mathrm{~mL}$ of acid ninhydrin, $2 \mathrm{~mL}$ of glacial acetic acid and $1 \mathrm{~mL}$ of filterate was heated in water bath at $100{ }^{\circ} \mathrm{C}$ for 1 hour and then transferred to ice bath following the addition of $4 \mathrm{~mL}$ of toluene. The reaction mixture was vortexed, chromophore having free proline was separated in test tube, kept at room temperature and the proline contents were measured at $520 \mathrm{~nm}$ on spectrophotometer (UV-1100). For blank, same procedure was used by using $2 \mathrm{~mL}$ of $3 \%$ aqueous sulphosalycylic acid. Following formula was used for proline determination.

\subsubsection{Soluble Sugars}

$$
\mu \text { moles } \frac{\text { proline }}{\mathrm{g}} \text { fresh weight }=\frac{\mu \mathrm{g} \text { proline } / \mathrm{mL} \times \mathrm{mL} \text { of toluene }}{(115.5 \mu \mathrm{g} / \mathrm{mole}) / \mathrm{g} \text { sample } / 5}
$$

For the determination of soluble sugars, the procedure given by Yoshida et al. (1976) was followed. Anthrone reagent was made by mixing $1 \mathrm{~g}$ anthrone in $1 \mathrm{~L}$ conc. $\mathrm{H}_{2} \mathrm{SO}_{4}$. For the determination of soluble sugars, $0.1 \mathrm{~g}$ fresh plant material was boiled in $5 \mathrm{~mL}$ distilled water and the filtrate was diluted to $50 \mathrm{~mL}$ with distilled water. To $1 \mathrm{~mL}$ of the filtrate, $5 \mathrm{~mL}$ of anthrone reagent was added and heated at $90{ }^{\circ} \mathrm{C}$ for $20 \mathrm{~min}$. The soluble sugar contents were determined at $620 \mathrm{~nm}$ by using spectrophotometer (UV-1100). For standard curve, glucose series $(0,20,40,60,80$ and $100 \mu \mathrm{M})$ was used. 


\subsubsection{Total Free Amino Acids}

Total free amino acids in plant tissues were measured by the procedure of Hamilton and Van-Slyke (1943). 2\% ninhydrine and $10 \%$ pyridine solution were prepared in the distilled water. For the determination of total free amino acids, $1 \mathrm{~g}$ fresh plant sample was homogenised in $10 \mathrm{~mL}$ of phosphate buffer $(0.2 \mathrm{M}$ with $\mathrm{pH} 7.2)$. To 1 $\mathrm{mL}$ of the extract, $1 \mathrm{~mL}$ of pyridine $(10 \%)$ and $1 \mathrm{~mL}$ of ninhydrine $(2 \%)$ were mixed and heated at $100{ }^{\circ} \mathrm{C}$ in water bath for $30 \mathrm{~min}$. The volume was maintained $50 \mathrm{~mL}$ with distilled water and the absorbance was noted at $570 \mathrm{~nm}$ by using spectrophotometer (UV-1100). Following formula was used for calculating total free amino acid.

$$
\text { Total amino acid }(\mathrm{mg} / \mathrm{g} \text { fresh weight })=\frac{\text { Graph reading of sample } \times \text { Volume of sample } \times \text { Dilution factor }}{\text { Weight of the tissue } \times 1000}
$$

\subsubsection{Total Soluble Proteins}

The concentration of total soluble protein was determined by the method given by Bradford (1976). Phosphate buffer saline was prepared by mixing $2.7 \mathrm{mM} \mathrm{KCl}, 10 \mathrm{mM} \mathrm{Na}_{2} \mathrm{HPO}_{4}, 1.37 \mathrm{mM} \mathrm{NaCl}$ and $2 \mathrm{mM} \mathrm{KH}_{2} \mathrm{PO}_{4}$ and $\mathrm{pH} 7.2$ was maintained by using $\mathrm{HCl}$. The determination of total soluble protein was done by extracting the $0.5 \mathrm{~g}$ fresh plant material in phosphate buffer saline, centrifugation was done and the supernatant was collected. To equal volume of supernatant dye stock was dissolved, vortexed and kept in an incubator for $30 \mathrm{~min}$. The absorbance was noted at $595 \mathrm{~nm}$ by using spectrophotometer (UV-1100). The standard curve was drawn by using bovine serum albumin (BSA) of the range $\left(10\right.$ to $\left.50 \mu \mathrm{g} \mathrm{mL}^{-1}\right)$.

\subsection{Determination of Inorganic Osmolytes}

\subsubsection{Sodium, Potassium, Calcium $\left(\mathrm{Na}^{+}, \mathrm{K}^{+}, \mathrm{Ca}^{2+}\right)$}

The dried plant sample $(0.5 \mathrm{~g})$ was incubated in $5 \mathrm{~mL} \mathrm{H}_{2} \mathrm{SO}_{4}$ overnight and heated at $350{ }^{\circ} \mathrm{C}$ in the digestion block for $30 \mathrm{~min}$. The mixture was cooled; $1 \mathrm{~mL}$ of $\mathrm{H}_{2} \mathrm{O}_{2}$ was added and again heated for $20 \mathrm{~min}$. These steps were repeated until clear solution was obtained, filtered, and volume was maintained to $50 \mathrm{~mL}$ by using distilled water (Wolf, 1982). This extract was used for the determination of $\mathrm{Na}^{+}, \mathrm{K}^{+}, \mathrm{Ca}^{2+}$ ions by using flame photometer (Jenway PFP-7). For standard curve a series of standards (10,20 to $100 \mathrm{ppm}$ of $\mathrm{Na}^{+}, \mathrm{K}^{+}$and $\mathrm{Ca}^{2+}$ ) was prepared. The actual values were calculated by comparing the values from standard curve and from flame photometer.

\subsubsection{Phosphate $\left(\mathrm{PO}_{4}^{2-}\right)$}

The concentration of phosphate ions in plant tissues was determined by following the method of Yoshida (1976). Firstly, two reagents were prepared. For the preparation of molybdate-vanadate solution, $25 \mathrm{~g}$ ammonium molybdate was mixed in $500 \mathrm{~mL}$ of water, and $1.25 \mathrm{~g}$ of ammonium vanadate was mixed in $500 \mathrm{~mL}$ of $1 \mathrm{~N} \mathrm{HNO}_{3}$ separately, then equal volumes of two solutions were mixed together. For the preparation of nitric acid $(2 \mathrm{~N}), 10$ $\mathrm{mL}$ of concentrated $\mathrm{HNO}_{3}$ was mixed in $80 \mathrm{~mL}$ of distilled water. The phosphate content was determined by boiling $0.5 \mathrm{~g}$ dried plant sample in $5 \mathrm{~mL}$ distilled water for $1 \mathrm{~h}$, filtered and $50 \mathrm{~mL}$ volume was prepared by using distilled water. $1 \mathrm{~mL}$ of extract was mixed with $2 \mathrm{~mL}$ of $2 \mathrm{~N} \mathrm{HNO}_{3}$, volume was maintained to $4 \mathrm{~mL}$ with distilled water, $1 \mathrm{~mL}$ of molybdate-vanadate reagent was added and the mixture was diluted to $10 \mathrm{~mL}$ with distilled water, vortexed, allowed to stand for $20 \mathrm{~min}$ and absorbance was noted at $420 \mathrm{~nm}$ by using spectrophotometer (UV-1100). For standard curve, stock solution of $25 \mathrm{mg} / \mathrm{L} \mathrm{PO}_{4}{ }^{3-}$ was prepared by mixing 0.11 $\mathrm{g}$ monobasic phosphate $\left(\mathrm{KH}_{2} \mathrm{PO}_{4}\right)$ in $1 \mathrm{~L}$ distilled water and standard series was prepared by mixing 1, 2, 3, 4, 5 and $6 \mathrm{~mL}$ of $25 \mathrm{mg} / \mathrm{L} \mathrm{PO}_{4}{ }^{3-}$ and diluted to $8 \mathrm{~mL}$ with distilled water.

\subsubsection{Nitrate $\left(\mathrm{NO}_{3}{ }^{-}\right)$}

For the determination of nitrate contents a procedure proposed by Kowalenko and Lowe (1973) was used. The reagents were prepared. For the preparation of $0.01 \%$ TCA, $0.1 \%$ CTA stock was prepared. For this purpose, $0.247 \mathrm{~g}$ of chromotropic acid disodium salt (CTA) was dissolved in $100 \mathrm{~mL}$ of conc. $\mathrm{H}_{2} \mathrm{SO}_{4}$. Then $10 \mathrm{~mL}$ of CTA stock was diluted to $100 \mathrm{~mL}$ with $\mathrm{H}_{2} \mathrm{SO}_{4}$ for the preparation of $0.01 \%$ TCA. For the determination of nitrate contents, $0.5 \mathrm{~g}$ dried plant sample was boiled in $5 \mathrm{~mL}$ of distilled water for $1 \mathrm{~h}$, filtered and diluted to $50 \mathrm{~mL}$ by using distilled water. $3 \mathrm{~mL}$ extract was mixed with $7 \mathrm{~mL}$ of working CTA solution, vortexed and absorbance was noted at $430 \mathrm{~nm}$ after $20 \mathrm{~min}$ by using spectrophotometer (UV-1100). Water was used for blank. For standard, $0.7216 \mathrm{~g}$ of $\mathrm{KNO}_{3}$ was dissolved in $1 \mathrm{~L}$ distilled water for the preparation of $100 \mathrm{mg} / \mathrm{L} \mathrm{NO}_{3}{ }^{-}$stock solution, then a graded series $\left(10,20,30,40,50\right.$ and $\left.100 \mathrm{mg} / \mathrm{L} \mathrm{NO}_{3}{ }^{-}\right)$was prepared by diluting the stock solution.

\subsubsection{Sulfate $\left(\mathrm{SO}_{4}{ }^{2-}\right)$}

For the determination of sulfate contents in plant sample, a procedure given by Tendon (1993) was used. Firstly, two reagents barium chloride/polyvinyl alcohol and acid mixture were prepared. For the preparation of barium 
chloride/polyvinyl alcohol, $60 \mathrm{~g}$ of $\mathrm{BaCl}_{2} \cdot 2 \mathrm{H}_{2} \mathrm{O}$ was dissolved in $500 \mathrm{~mL}$ distilled/deionized water and $2 \mathrm{~g}$ polyvinyl alcohol was dissolved in $400 \mathrm{~mL}$ distilled water separately. The two solutions were mixed, filtered and volume was maintained to $1 \mathrm{~L}$ by using distilled water. Acid mixture was prepared by mixing $50 \mathrm{~mL}$ of glacial acetic acid, $20 \mathrm{~mL}$ of $85 \%$ orthophosphoric acid, $6 \mathrm{~mL}$ of concentrated sulfuric acid/water (with a ratio of 1:1000) and $800 \mathrm{~mL}$ of distilled water was thoroughly mixed and diluted to $1 \mathrm{~L}$ by using distilled water. Sulfate contents were determined by $5 \mathrm{~mL}$ of the sample solution, $5 \mathrm{~mL}$ of the acid mixture and $5 \mathrm{~mL}$ of barium chloride/polyvinyl alcohol was mixed, allowed to stand for 30 seconds and aliquot was collected for the determination of absorbance at $420 \mathrm{~nm}$ by using spectrophotometer (UV-1100).

\subsection{Statistical Analysis}

The experimental design was completely randomized (CRD) with three factor factorial arrangement. The data was analyzed statistically by analysis of variance technique (ANOVA) (Steel \& Torrie, 1986) by using Co-Stat software (CoHort Software, 2003, Monterey, California). Microsoft excel was used for the preparation of figures.

\section{Results}

\subsection{Organic Osmolytes}

\subsubsection{Glycine Betaine}

Results revealed that salt stress caused the accumulation of glycine betaine contents in both maize cultivars. The maximum concentration of glycine betaine was found at $75 \mathrm{mM}$ salt level in all tissues of maize plants (root, shoot, leaf). It was evident from statistically significant $\mathrm{V} \times \mathrm{Sa}$ interactive effect in shoot and root while in leaf this interaction was non-significant (Table 1). Application of sulfur improved the glycine betaine contents in both maize varieties. It was shown by significant $\mathrm{V} \times \mathrm{Sa}$ interactive effect in leaf. However, in leaf and shoot this interaction was found non-significant (Table 1). Sulfur at $40 \mathrm{mM}$ level highly improved the glycine betaine contents in root, shoot and leaf of both maize varieties at all salt levels $(25,75 \mathrm{mM})$ (Figure 1). It was evident by significant $\mathrm{Sa} \times \mathrm{S}$ interaction. However, in root and leaf $\mathrm{Sa} \times \mathrm{S}$ interaction was found non-significant (Table 1). The order of decreasing the glycine betaine contents in plant tissue was leaf $>$ shoot $>$ root. Salt tolerant variety (Agaitti, 2003) accumulated high glycine betaine contents as compared to salt sensitive variety (Pak Afgoi, 2003).

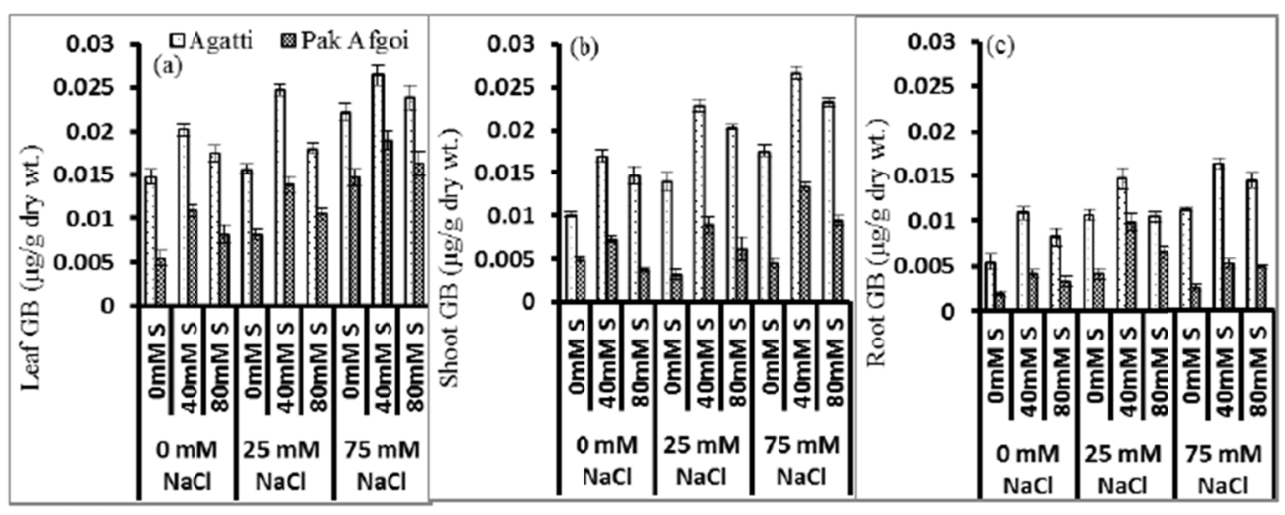

Figure 1. Effect of different levels of sulfur (S) on glycine betaine content in leaf (a) shoot (b) root (c) of different maize (Zea mays L.) cultivars under saline conditions 
Table 1. Mean squares from analysis of variance (ANOVA) of the data for glycine betaine and proline contents of maize subjected to different levels of salinity and sulfur

\begin{tabular}{llllllll}
\hline SOV & Df & Leaf GB & Shoot GB & Root GB & Leaf Proline & Shoot Proline & Root Proline \\
\hline Variety (V) & 1 & $9.68 \mathrm{e}-4 * * *$ & $0.0018 * * *$ & $6.04 \mathrm{e}-4 * * *$ & $1.65 \mathrm{e}-6 * * *$ & $2.03 \mathrm{e}-6 * * *$ & $1.24 \mathrm{e}-6 * 6^{* *}$ \\
Salinity (Sa) & 2 & $2.72 \mathrm{e}-4 * * *$ & $1.70 \mathrm{e}-4 * * *$ & $7.96 \mathrm{e}-5 * * *$ & $6.41 \mathrm{e}-7 * * *$ & $1.14 \mathrm{e}-7 * * *$ & $2.34 \mathrm{e}-7 * * *$ \\
Sulfur (S) & 2 & $1.46 \mathrm{e}-4 * * *$ & $2.15 \mathrm{e}-4 * * *$ & $7.96 \mathrm{e}-5 * * *$ & $5.52 \mathrm{e}-7 * * *$ & $1.36 \mathrm{e}-7 * * *$ & $2.75 \mathrm{e}-7 * * *$ \\
$\mathrm{~V} \times \mathrm{Sa}$ & 2 & $3.71 \mathrm{e}-6 \mathrm{~ns}$ & $3.08 \mathrm{e}-5 * * *$ & $3.49 \mathrm{e}-5 * * *$ & $1.04 \mathrm{e}-38 \mathrm{~ns}$ & $7.47 \mathrm{e}-9 \mathrm{~ns}$ & $3.86 \mathrm{e}-8 * * *$ \\
$\mathrm{~V} \times \mathrm{S}$ & 2 & $1.85 \mathrm{e}-6 \mathrm{~ns}$ & $1.38 \mathrm{e}-5 * * *$ & $2.62 \mathrm{e}-6 \mathrm{~ns}$ & $1.04 \mathrm{e}-38 \mathrm{~ns}$ & $7.29 \mathrm{e}-10 \mathrm{~ns}$ & $5.37 \mathrm{e}-9 *$ \\
$\mathrm{Sa} \times \mathrm{S}$ & 4 & $4.71 \mathrm{e}-6 \mathrm{~ns}$ & $8.98 \mathrm{e}-6 * * *$ & $3.08 \mathrm{e}-6 \mathrm{~ns}$ & $1.25 \mathrm{e}-8 *$ & $2.56 \mathrm{e}-9 \mathrm{~ns}$ & $1.24 \mathrm{e}-8 * * *$ \\
$\mathrm{~V} \times \mathrm{Sa} \times \mathrm{S}$ & 4 & $1.85 \mathrm{e}-6 \mathrm{~ns}$ & $2.55 \mathrm{e}-6 \mathrm{~ns}$ & $2.76 \mathrm{e}-6 \mathrm{~ns}$ & $3.32 \mathrm{e}-38 \mathrm{~ns}$ & $1.79 \mathrm{e}-9 \mathrm{~ns}$ & $6.40 \mathrm{e}-9 * *$ \\
Error & 36 & $2.60 \mathrm{E}-06$ & $1.52 \mathrm{E}-06$ & $1.37 \mathrm{E}-06$ & $4.46 \mathrm{E}-09$ & $3.07 \mathrm{E}-09$ & $1.36 \mathrm{E}-09$ \\
\hline
\end{tabular}

Note. ${ }^{*}, * *, * * *=$ significant at $0.05,0.01$ and 0.001 levels, respectively. $\mathrm{ns}=$ non-significant.

Abbreviation: Exponent (e), GB = Glycine Betaine.

\subsubsection{Proline}

Statistical analysis has shown that proline contents were increased by increasing the salinity in both maize cultivars. It was evident from significant $\mathrm{V} \times \mathrm{Sa}$ interaction for root, while in shoot and leaf $\mathrm{V} \times \mathrm{Sa}$ interaction was found non-significant (Table 1). At $75 \mathrm{mM}$ salt level proline contents were high in both studied maize cultivars. The decreasing order of proline contents was leaves $>$ shoot $>$ root (Figure 2). Application of sulfur at $80 \mathrm{mM}$ level did not much improve the proline contents significantly. However sulfur application was synergistic to the salinity effect in accumulating the proline contents in maize organs. It was shown by significant $\mathrm{Sa} \times \mathrm{S}$ interaction for leaf and root while in shoot $\mathrm{Sa} \times \mathrm{S}$ interaction was found non-significant (Table 1). Pak Afgoi (2003) accumulated low proline contents as compared to Agaitti (2003). This variation in variety is shown by significant $\mathrm{V} \times \mathrm{Sa} \times \mathrm{S}$ interaction for maize root while for shoot and root $\mathrm{V} \times \mathrm{Sa} \times \mathrm{S}$ interactive effect was found non-significant indicating that sulfur application decreased the proline contents in maize plants in both varieties at all levels of treatment $(40,60 \mathrm{mM})$ (Figure 2).

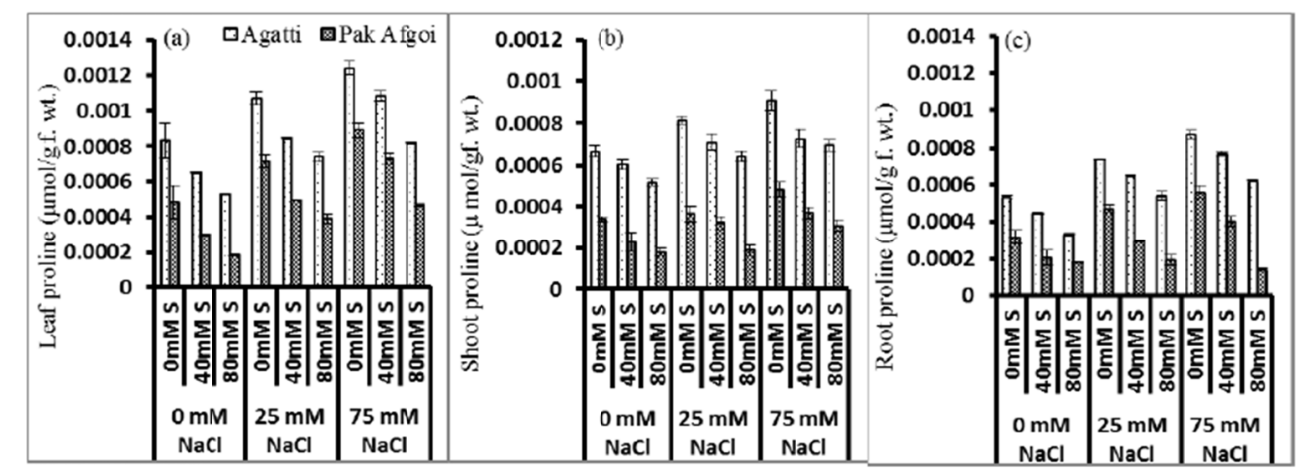

Figure 2. Effect of different levels of sulfur (S) on proline content in leaf (a) shoot (b) root (c) of different maize (Zea mays L.) cultivars under saline conditions

\subsubsection{Total Soluble Sugar}

A marked increase in total soluble sugar contents by salt application was found in both studied maize cultivars. It was shown by statistically significant $\mathrm{V} \times \mathrm{Sa}$ interaction for leaf, shoot and root (Table 2). 


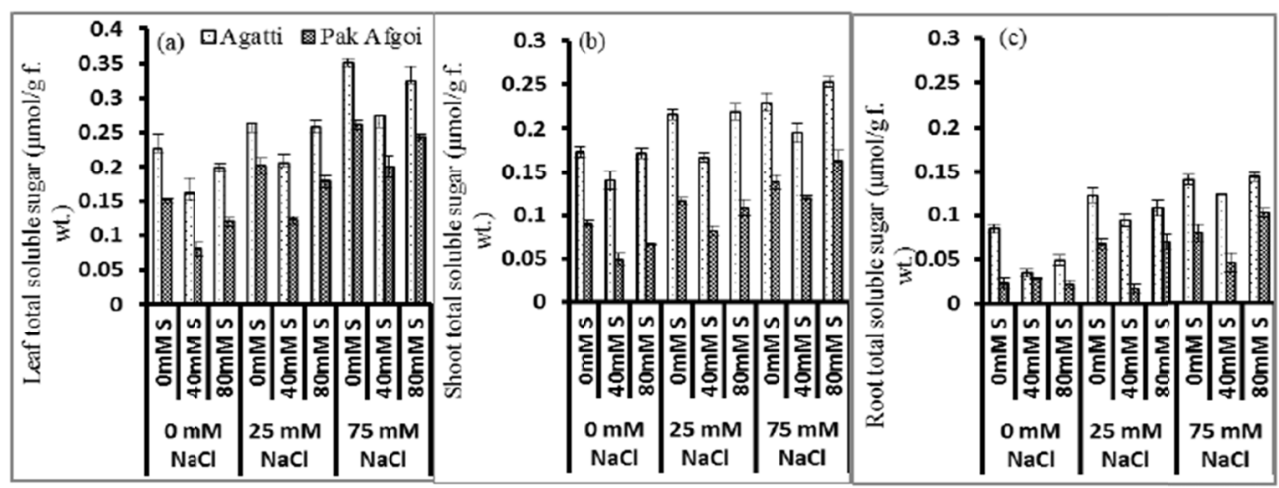

Figure 3. Effect of different levels of sulfur (S) on total soluble sugar content in leaf (a) shoot (b) root (c) of different maize (Zea mays L.) cultivars under saline conditions

Sulfur application $(40,80 \mathrm{mM})$ significantly improved the soluble sugar contents in all studied maize organs at all levels of salinity. It was shown by significant $\mathrm{Sa} \times \mathrm{S}$ interaction for shoot and root, however, for leaf $\mathrm{Sa} \times \mathrm{S}$ interaction was found non-significant (Table 2). Sulfur at $40 \mathrm{mM}$ level significantly improved the total soluble sugar contents in both studied maize cultivars (Figure 3). It was evident form statistically significant $\mathrm{V} \times \mathrm{Sa} \times \mathrm{S}$ interaction in both maize varieties (Table 2).

Table 2. Mean squares from analysis of variance (ANOVA) of the data for total soluble sugars and total soluble protein contents of maize subjected to different levels of salinity and sulfur

\begin{tabular}{llllllll}
\hline SOV & df & Leaf TSS & Shoot TSS & Root TSS & Leaf TSP & Shoot TSP & Root TSP \\
\hline Variety (V) & 1 & $0.083 * * *$ & $0.11 * * *$ & $0.033 * * *$ & $0.021 * * *$ & $0.0053 * * *$ & $0.0042 * * *$ \\
Salinity (Sa) & 2 & $0.065 * * *$ & $0.021 * * *$ & $0.019 * * *$ & $0.0038 * * *$ & $0.0015 * * *$ & $8.14 \mathrm{e}-4 * * *$ \\
Sulfur (S) & 2 & $0.022 * * *$ & $0.0081 * * *$ & $0.0044 * * *$ & $0.0077 * * *$ & $0.0019 * * *$ & $0.0043 * * *$ \\
V $\times$ Sa & 2 & $5.39 \mathrm{e}-5 \mathrm{~ns}$ & $1.91 \mathrm{e}-4 \mathrm{~ns}$ & $0.0011^{* *}$ & $4.5 \mathrm{e}-4 * * *$ & $8.79 \mathrm{e}-5 *$ & $1.93 \mathrm{e}-4 *$ \\
$\mathrm{~V} \times \mathrm{S}$ & 2 & $2.63 \mathrm{e}-5 \mathrm{~ns}$ & $3.78 \mathrm{e}-4 \mathrm{~ns}$ & $6.85 \mathrm{e}-4 *$ & $1.71 \mathrm{e}-34 \mathrm{~ns}$ & $1.67 \mathrm{e}-5 \mathrm{~ns}$ & $7.91 \mathrm{e}-5 \mathrm{~ns}$ \\
$\mathrm{Sa} \times \mathrm{S}$ & 4 & $1.18 \mathrm{e}-4 \mathrm{~ns}$ & $6.0033 \mathrm{e}-4 *$ & $6.84 \mathrm{e}-4 * *$ & $1.44 \mathrm{e}-4 *$ & $1.07 \mathrm{e}-4 * * *$ & $9.33 \mathrm{e}-5 \mathrm{~ns}$ \\
$\mathrm{~V} \times \mathrm{Sa} \times \mathrm{S}$ & 4 & $1.45 \mathrm{e}-4 \mathrm{~ns}$ & $9.22 \mathrm{e}-5 \mathrm{~ns}$ & $7.72 \mathrm{e}-4 * *$ & $2.41 \mathrm{e}-34 \mathrm{~ns}$ & $5.55 \mathrm{e}-5 *$ & $6.88 \mathrm{e}-5 \mathrm{~ns}$ \\
Error & 36 & $4.09 \mathrm{E}-04$ & $1.72 \mathrm{E}-04$ & $1.41 \mathrm{E}-04$ & $5.14 \mathrm{E}-05$ & $1.78 \mathrm{E}-05$ & $5.61 \mathrm{E}-05$ \\
\hline
\end{tabular}

Note. ${ }^{*}, * *, * * *=$ significant at $0.05,0.01$ and 0.001 levels, respectively. $\mathrm{ns}=$ non-significant.

Abbreviation: Exponent (e), TSS = Total Soluble Sugars, TSP = Total Soluble Protein .

\subsubsection{Total Soluble Protein}

Exposure to maize varieties to salinity increased the total soluble protein contents in both studied maize varieties. It was indicated by statistically significant $\mathrm{V} \times \mathrm{Sa}$ interaction (Table 2). At $75 \mathrm{mM}$ salt level total soluble protein contents were high (Figure 4). The exogenous application of sulfur (40, $80 \mathrm{mM}$ ) improved the total soluble protein contents in both maize genotypes. However $40 \mathrm{mM}$ sulfur level was found appropriate in improving the total soluble protein contents (Figure 4). It was shown by statistically significant $\mathrm{Sa} \times \mathrm{S}$ interaction for leaf and shoot and non-significant in root (Table 2). Agaitti (2003) accumulated more the total soluble protein contents as compared to Pak Afgoi (2003). It was revealed form significant $\mathrm{V} \times \mathrm{Sa} \times \mathrm{S}$ interaction for maize shoot. The maximum accumulation of the total soluble protein contents was found in maize leaves which decreased in shoot and root respectively (Figure 4). 


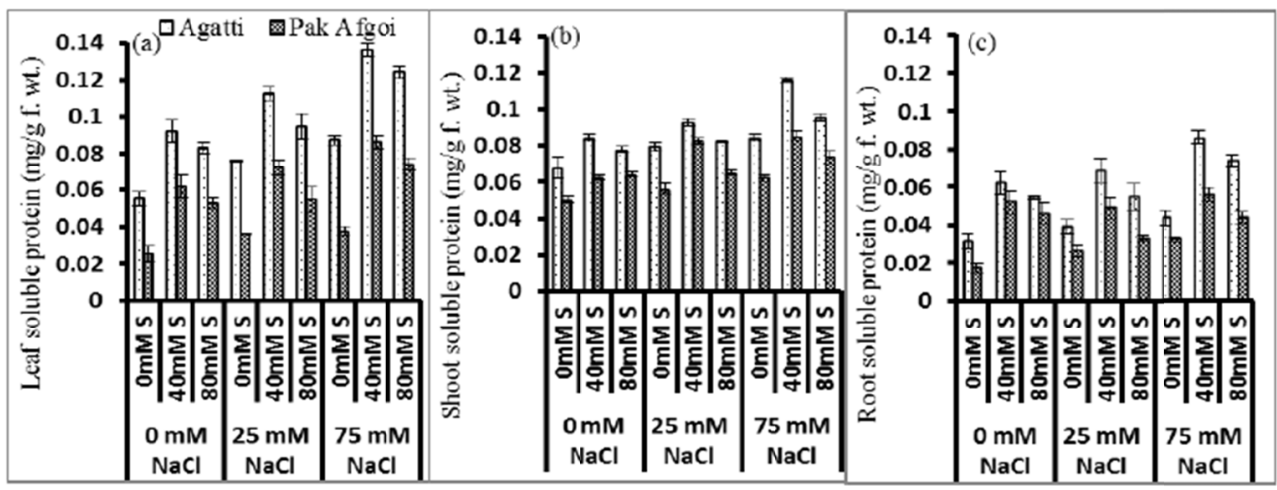

Figure 4. Effect of different levels of sulfur (S) on total soluble protein content in leaf (a) shoot (b) root (c) of different maize (Zea mays L.) cultivars under saline conditions

\subsubsection{Total Free Amino Acid}

Results revealed that salinity caused increase in total free amino acid contents in both studied maize cultivars. Maximum improvement in total free amino acid contents was found at $75 \mathrm{mM}$ salt level (Figure 5). The application of sulfur $(40 \mathrm{mM})$ improved the total free amino acid contents in all studied maize organs. It was shown by statistically significant $\mathrm{Sa} \times \mathrm{S}$ interaction for leaf and root and non-significant for shoot (Table 3 ).

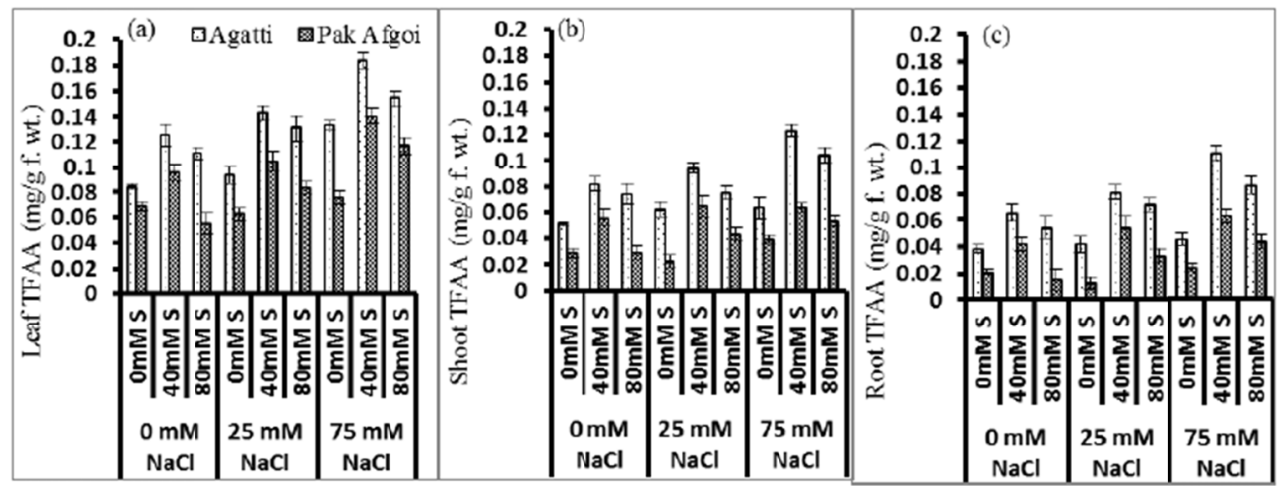

Figure 5. Effect of different levels of sulfur (S) on total free amino acid content in leaf (a) shoot (b) root (c) of different maize (Zea mays L.) cultivars under saline conditions

Salt tolerant maize cultivar (Agaitti, 2003) accumulated high total free amino acid contents as compared to salt sensitive variety (Pak Afgoi, 2003). It was evident form significant $\mathrm{V} \times \mathrm{Sa} \times \mathrm{S}$ interaction (Table 3). The order of accumulation of total free amino acid contents in maize organs was leaf $>$ shoot $>$ root (Figure 5).

Table 3. Mean squares from analysis of variance (ANOVA) of the data for total soluble sugars and total free amino acid contents of maize subjected to different levels of salinity and sulfur

\begin{tabular}{lllll}
\hline SOV & df & Leaf TFA & Shoot TFA & Root TFA \\
\hline Variety (V) & 1 & $0.021 * * *$ & $0.018 * * *$ & $0.013^{* * *}$ \\
Salinity (Sa) & 2 & $0.0092 * * *$ & $0.0020 * * *$ & $0.0023 * * *$ \\
Sulfur (S) & 2 & $0.0093 * * *$ & $0.0058 * * *$ & $0.0067 * * *$ \\
$\mathrm{~V} \times \mathrm{Sa}$ & 2 & $1.74 \mathrm{e}-4 \mathrm{~ns}$ & $2.42 \mathrm{e}-4 \mathrm{~ns}$ & $1.23 \mathrm{e}-4 \mathrm{~ns}$ \\
$\mathrm{~V} \times \mathrm{S}$ & 2 & $1.92 \mathrm{e}-4 \mathrm{~ns}$ & $2.30 \mathrm{e}-4 \mathrm{~ns}$ & $3.24 \mathrm{e}-4 \mathrm{~ns}$ \\
$\mathrm{Sa} \times \mathrm{S}$ & 4 & $3.61 \mathrm{e}-4 *$ & $1.14 \mathrm{e}-4 \mathrm{~ns}$ & $3.98 \mathrm{e}-4 * *$ \\
$\mathrm{~V} \times \mathrm{Sa} \times \mathrm{S}$ & 4 & $3.49 \mathrm{e}-4 *$ & $2.62 \mathrm{e}-4 *$ & $8.98 \mathrm{e}-5 \mathrm{~ns}$ \\
Error & 36 & $1.20 \mathrm{E}-04$ & $8.97 \mathrm{E}-05$ & $1.01 \mathrm{E}-04$ \\
\hline
\end{tabular}

Note. ${ }^{*},{ }^{* *},{ }^{* * *}=$ significant at $0.05,0.01$ and 0.001 levels, respectively. $\mathrm{ns}=$ non-significant.

Abbreviation: Exponent (e), TFF $=$ Total Free Amino Acid. 


\subsection{Inorganic Osmolytes}

\subsubsection{Sodium $\left(\mathrm{Na}^{+}\right)$}

Results revealed that salinity increased the sodium $\left(\mathrm{Na}^{+}\right)$contents in both studied maize cultivars. It was shown by statistically significant $\mathrm{V} \times \mathrm{Sa}$ interaction for shoot. However, in root and leaf, $\mathrm{V} \times \mathrm{Sa}$ interaction was found non-significant (Table 4). At $75 \mathrm{mM}$ salt level, sodium $\left(\mathrm{Na}^{+}\right)$contents very found very high (Figure 6).

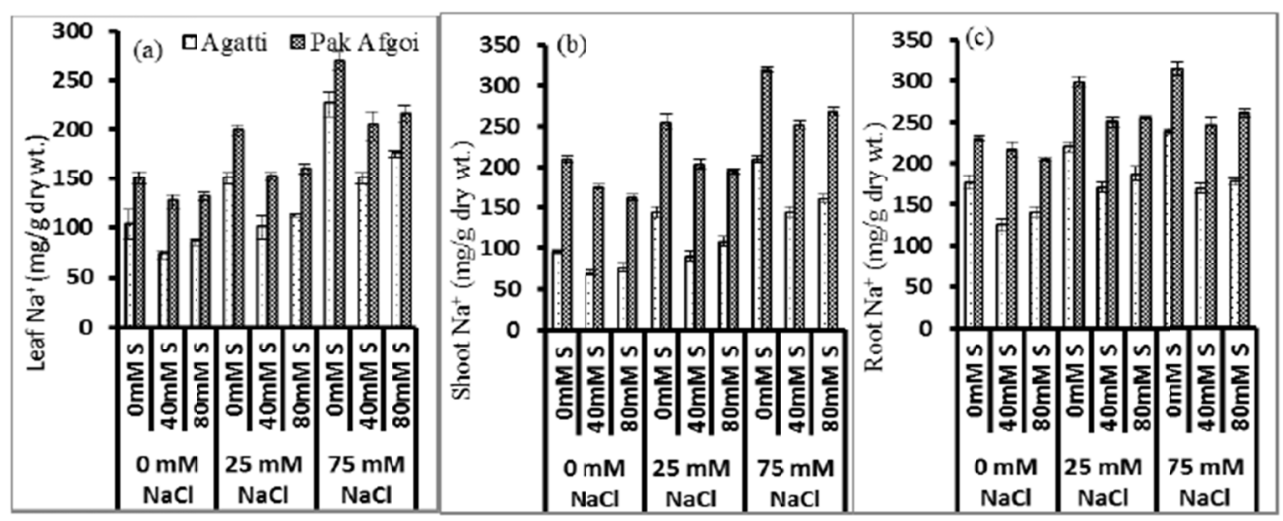

Figure 6. Effect of different levels of sulfur (S) on $\mathrm{Na}^{+}$content in leaf (a) shoot (b) root (c) of different maize (Zea mays L.) cultivars under saline conditions

The application of sulfur $(40,80 \mathrm{mM})$ significantly lowered the sodium $\left(\mathrm{Na}^{+}\right)$contents in all studied maize organs at both studied salt levels $(25,75 \mathrm{mM})$. It was evident from statistically significant $\mathrm{Sa} \times \mathrm{S}$ interactive effect (Table 4). Leaf had high sodium contents as compared to shoot and root. Moreover, sulfur at $40 \mathrm{mM}$ level lowered the sodium $\left(\mathrm{Na}^{+}\right)$contents in Agaitti (2003) as compared to Pak Afgoi (2003) (Figure 6).

Table 4. Mean squares from analysis of variance (ANOVA) of the data for sodium $\left(\mathrm{Na}^{+}\right)$and potassium $\left(\mathrm{K}^{+}\right)$ contents of maize subjected to different levels of salinity and sulfur

\begin{tabular}{llllllll}
\hline SOV & df & Leaf Na $^{+}$ & ${\text {Shoot } \mathrm{Na}^{+}}^{*}$ & ${\text { Root } \mathrm{Na}^{+}}^{*}$ & Leaf $^{+}$ & Shoot $^{+}$ & Root $^{+}$ \\
\hline Variety (V) & 1 & $30706.87 * * *$ & $147796.33 * *$ & $75298.84 * * *$ & $285871.13 * * *$ & $322634.74 * * *$ & $29 * * *$ \\
Salinity (Sa) & 2 & $41258.27 * * *$ & $41078.29 * * *$ & $15390.76^{* * *}$ & $101395.78 * * *$ & $87805.52 * * *$ & $58592.55 * * *$ \\
Sulfur (S) & 2 & $11438.75 * * *$ & $13295.002 * * *$ & $13069.02 * * *$ & $54869.21 * * *$ & $68620.39 * * *$ & $43139.16 * * *$ \\
$\mathrm{~V} \times \mathrm{Sa}$ & 2 & $19.27 \mathrm{~ns}$ & $66.51 \mathrm{~ns}$ & $82.94 \mathrm{~ns}$ & $554.57 * *$ & $3603.46 *$ & $3531.72 *$ \\
$\mathrm{~V} \times \mathrm{S}$ & 2 & $93.05 \mathrm{~ns}$ & $438.54 * *$ & $224.82 \mathrm{~ns}$ & $209.68 \mathrm{~ns}$ & $170.12 \mathrm{~ns}$ & $56.72 \mathrm{~ns}$ \\
$\mathrm{Sa} \times \mathrm{S}$ & 4 & $776.68 * *$ & $551.33 * * *$ & $531.58 * *$ & $183.66 \mathrm{~ns}$ & $681.06 \mathrm{~ns}$ & $657.90 \mathrm{~ns}$ \\
$\mathrm{~V} \times \mathrm{Sa} \times \mathrm{S}$ & 4 & $10.05 \mathrm{~ns}$ & $108.38 \mathrm{~ns}$ & $229.44 \mathrm{~ns}$ & $116.46 \mathrm{~ns}$ & $382.26 \mathrm{~ns}$ & $1077.44 \mathrm{~ns}$ \\
Error & 36 & 194.22 & 78.64 & 120.17 & 100.95 & 844.67 & 810.17 \\
\hline
\end{tabular}

Note. ${ }^{*}, * *, * * *=$ significant at $0.05,0.01$ and 0.001 levels, respectively. $\mathrm{ns}=$ non-significant.

Abbreviation: Exponent (e), $\mathrm{Na}^{+}=$Sodium, $\mathrm{K}^{+}=$Potassium.

\subsubsection{Potassium $\left(\mathrm{K}^{+}\right)$}

Statistical analysis revealed that salinity reduced the potassium $\left(\mathrm{K}^{+}\right)$contents in shoot, leaf and root of both maize cultivars (Figure 7). It was evident form statistically significant $\mathrm{V} \times \mathrm{Sa}$ interaction (Table 4). At $40 \mathrm{mM}$ sulfur level, potassium $\left(\mathrm{K}^{+}\right)$contents very improved in both varieties (Agaitti, 2003; Pak Afgoi, 2003) (Figure 7). However, both levels of sulfur $(40,80 \mathrm{mM})$ improved the potassium $\left(\mathrm{K}^{+}\right)$contents at both levels of salt treatment $(25,75 \mathrm{mM})$ in both maize cultivars. Maximum potassium $\left(\mathrm{K}^{+}\right)$contents were found in leaf then shoot and root respectively (Figure 7). 


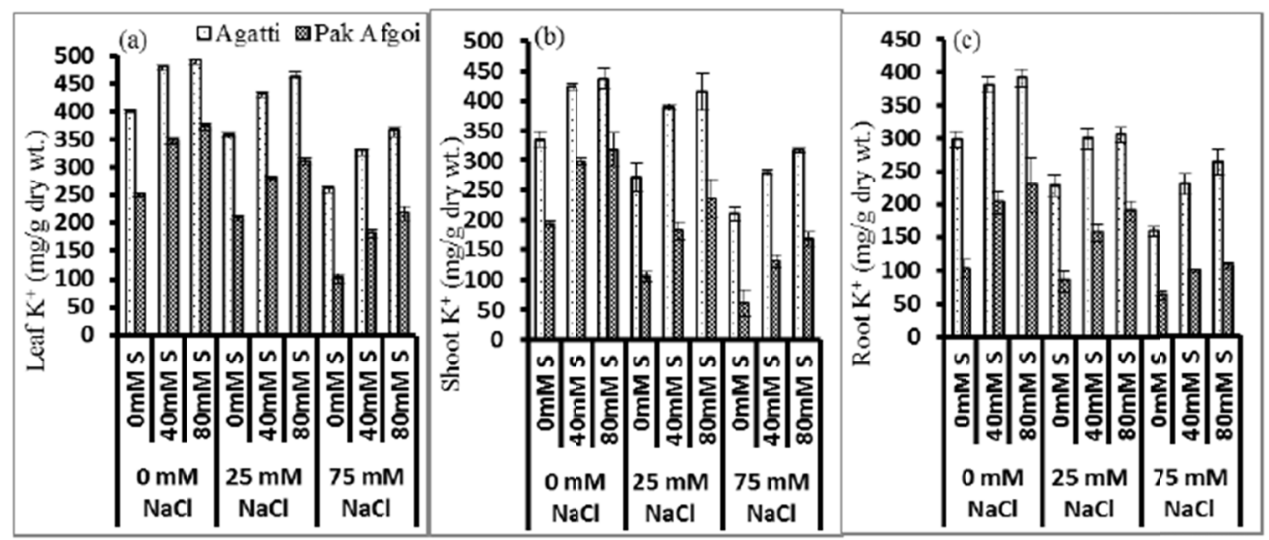

Figure 7. Effect of different levels of sulfur (S) on $\mathrm{K}^{+}$content in leaf (a) shoot (b) root (c) of different maize (Zea mays L.) cultivars under saline conditions

\subsubsection{Calcium $\left(\mathrm{Ca}^{2+}\right)$}

Calcium $\left(\mathrm{Ca}^{2+}\right)$ contents were reduced by salt application $(25,75 \mathrm{mM})$ in all studied maize organs (leaf, shoot, root) (Figure 8). It was evident from significant $\mathrm{V} \times \mathrm{Sa}$ interaction for leaf and shoot, however in $\operatorname{root} \mathrm{V} \times \mathrm{Sa}$ interaction was found non-significant.

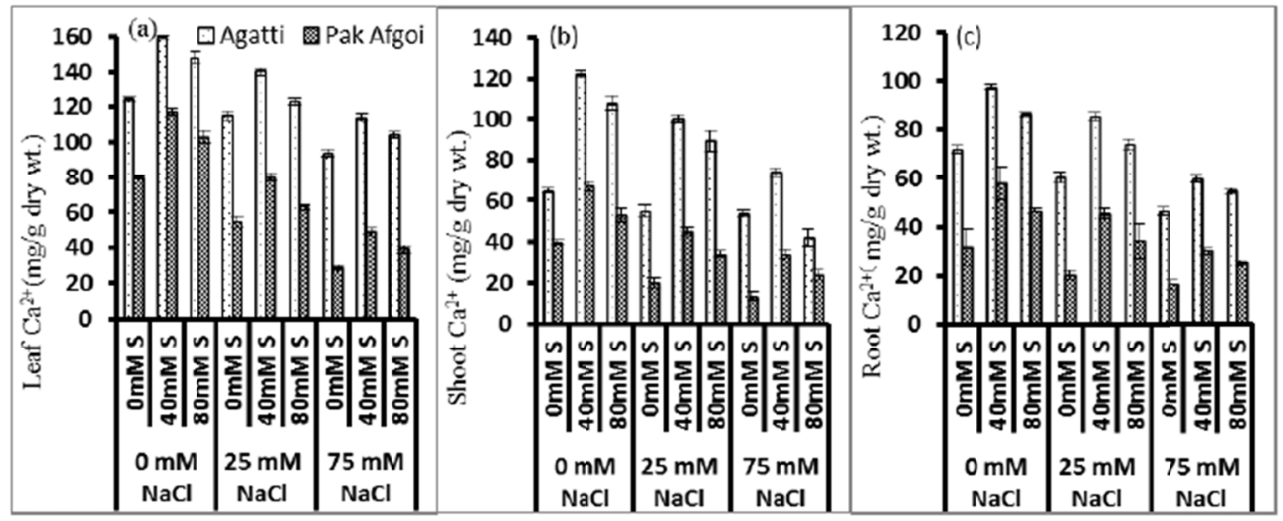

Figure 8. Effect of different levels of sulfur (S) on $\mathrm{Ca}^{2+}$ content in leaf (a) shoot (b) root (c) of different maize (Zea mays L.) cultivars under saline conditions

The exogenous application of sulfur $(40,80 \mathrm{mM})$ improved the calcium $\left(\mathrm{Ca}^{2+}\right)$ contents in both studied maize cultivars. It was shown by statistically significant $\mathrm{Sa} \times \mathrm{S}$ and $\mathrm{V} \times \mathrm{S}$ interaction in leaf and shoot while in root this interaction was found non-significant (Table 5). Also both levels of sulfur $(40,80 \mathrm{mM})$ lowered the toxic effects of salinity by improving the calcium $\left(\mathrm{Ca}^{2+}\right)$ contents at higher levels of salinity in salt tolerant (Agaitti, 2003) and salt sensitive (Pak Afgoi, 2003) cultivars (Figure 8). It was revealed from statistically significant $\mathrm{V} \times \mathrm{Sa} \times \mathrm{S}$ interaction (Table 5). 
Table 5. Mean squares from analysis of variance (ANOVA) of the data for calcium $\left(\mathrm{Ca}^{2+}\right)$ and $\mathrm{K}^{+} / \mathrm{Na}^{+}$ratio of maize subjected to different levels of salinity and sulfur

\begin{tabular}{|c|c|c|c|c|c|c|c|}
\hline SOV & $\mathrm{df}$ & Leaf $\mathrm{Ca}^{2+}$ & Shoot $\mathrm{Ca}^{2+}$ & Root $\mathrm{Ca}^{2+}$ & Leaf $\mathrm{K}^{+} / \mathrm{Na}^{+}$ratio & Shoot $\mathrm{K}^{+} / \mathrm{Na}^{+}$ratio & Root $\mathrm{K}^{+} / \mathrm{Na}^{+}$ratio \\
\hline Variety (V) & 1 & $42887.33 * * *$ & $23856.01 * * *$ & $18113.35 * * *$ & $55.23 * * *$ & $15.99 * * *$ & $7.34 * * *$ \\
\hline Salinity (Sa) & 2 & $11499.46 * * *$ & $5691.72 * * *$ & $3126.76 * * *$ & $31.44 * * *$ & $9.17 * * *$ & $3.61 * * *$ \\
\hline Sulfur (S) & 2 & $3336.05 * * *$ & $4811.67 * * *$ & $2128.28 * * *$ & $9.59 * * *$ & $3.52 * * *$ & $1.46 * * *$ \\
\hline $\mathrm{V} \times \mathrm{Sa}$ & 2 & $536.49 * * *$ & $317.62 * * *$ & $148.35 *$ & $4.30 * * *$ & $0.42 * * *$ & $0.25 * * *$ \\
\hline $\mathrm{V} \times \mathrm{S}$ & 2 & $1.24 \mathrm{~ns}$ & $314.35 * * *$ & $0.018 \mathrm{~ns}$ & $1.30 * *$ & $0.14 * *$ & $0.10 * * *$ \\
\hline $\mathrm{Sa} \times \mathrm{S}$ & 4 & $145.27 * * *$ & $399.34 * * *$ & $66.67 \mathrm{~ns}$ & $0.45 \mathrm{~ns}$ & $0.11 * *$ & $0.065 * *$ \\
\hline $\mathrm{V} \times \mathrm{Sa} \times \mathrm{S}$ & 4 & $1.24 \mathrm{~ns}$ & $295.96 * * *$ & $0.018 \mathrm{~ns}$ & $0.097 \mathrm{~ns}$ & $0.022 \mathrm{~ns}$ & $0.018 \mathrm{~ns}$ \\
\hline Error & 36 & 14.78 & 22.01 & 31.71 & 0.19 & 0.027 & 0.012 \\
\hline
\end{tabular}

Note. ${ }^{*}, * *, * * *=$ significant at $0.05,0.01$ and 0.001 levels, respectively. $\mathrm{ns}=$ non-significant.

Abbreviation: Exponent (e), $\mathrm{Ca}^{2+}=$ Calcium, $\mathrm{Na}^{+}=$Sodium, $\mathrm{K}^{+}=$Potassium .

\section{$3.2 .4 \mathrm{~K}^{+} / \mathrm{Na}^{+}$}

Salinity caused the reduction in $\mathrm{K}^{+} / \mathrm{Na}^{+}$ratio in salt tolerant and salt sensitive maize cultivars. Maximum reduction in $\mathrm{K}^{+} / \mathrm{Na}^{+}$ratio was found at $75 \mathrm{mM}$ salt applied (Figure 9). However, sulfur application significantly improved the $\mathrm{K}^{+} / \mathrm{Na}^{+}$ratio in both varieties. It was evident from statistically significant $\mathrm{V} \times \mathrm{S}$ interaction for all studied maize organs (Table 5). Sulfur also developed salt tolerance in maize plants by reducing the toxic effect of salinity. A statistically significant $\mathrm{Sa} \times \mathrm{S}$ interaction in shoot and root, revealed this fact (Table 5). Salt tolerant variety (Agaitti, 2003) responded well to sulfur application by improving the $\mathrm{K}^{+} / \mathrm{Na}^{+}$ratio in comparison to salt sensitive variety (Pak Afgoi, 2003) (Figure 9).

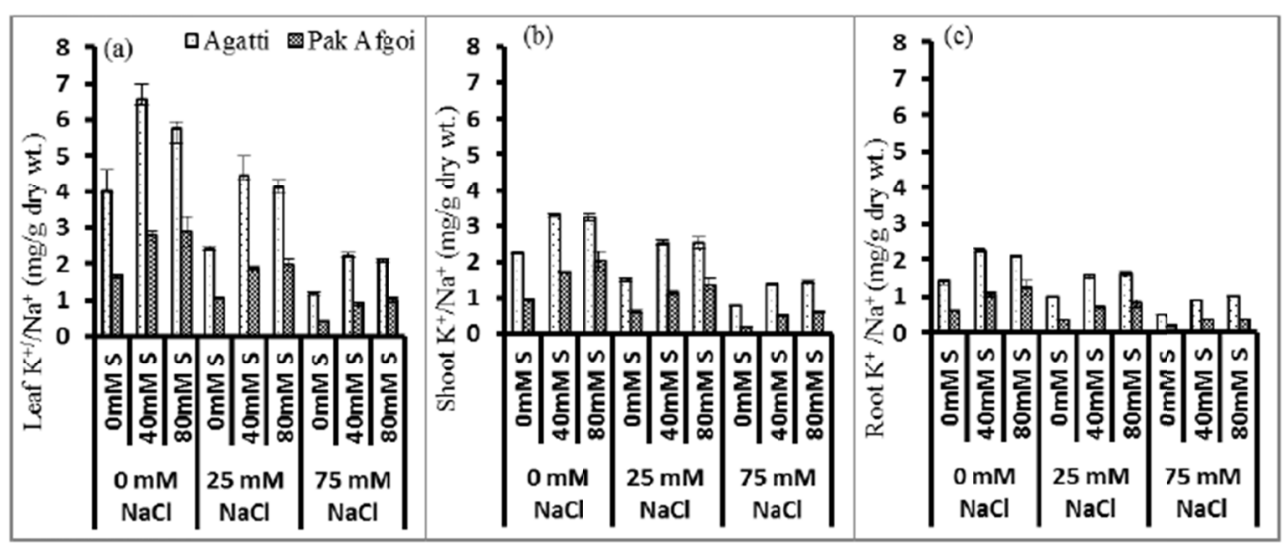

Figure 9. Effect of different levels of sulfur (S) on $\mathrm{K}^{+} / \mathrm{Na}^{+}$content in leaf (a) shoot (b) root (c) of different maize (Zea mays L.) cultivars under saline conditions

\subsection{5 $\mathrm{Ca}^{2+} / \mathrm{Na}^{+}$}

Results showed that salt stress $(25,75 \mathrm{mM})$ reduced the $\mathrm{Ca}^{2+} / \mathrm{Na}^{+}$ratio in maize plants. It was revealed from statistically significant $\mathrm{V} \times \mathrm{Sa}$ interaction in leaf, shoot and root (Table 6). The application of sulfur not only improved the $\mathrm{Ca}^{2+} \mathrm{Na}^{+}$ratio, but also developed salt tolerance in both maize cultivars at all levels of salinity (25, $75 \mathrm{mM}$ ). This fact is evident from statistically significant $\mathrm{Sa} \times \mathrm{S}$ interaction, while $\mathrm{V} \times \mathrm{Sa} \times \mathrm{S}$ interaction was significant only for maize root (Table 6). Agaitti (2003) accumulated high $\mathrm{Ca}^{2+} / \mathrm{Na}^{+}$ratio as compared to Pak Afgoi 2003 (Figure 10). 


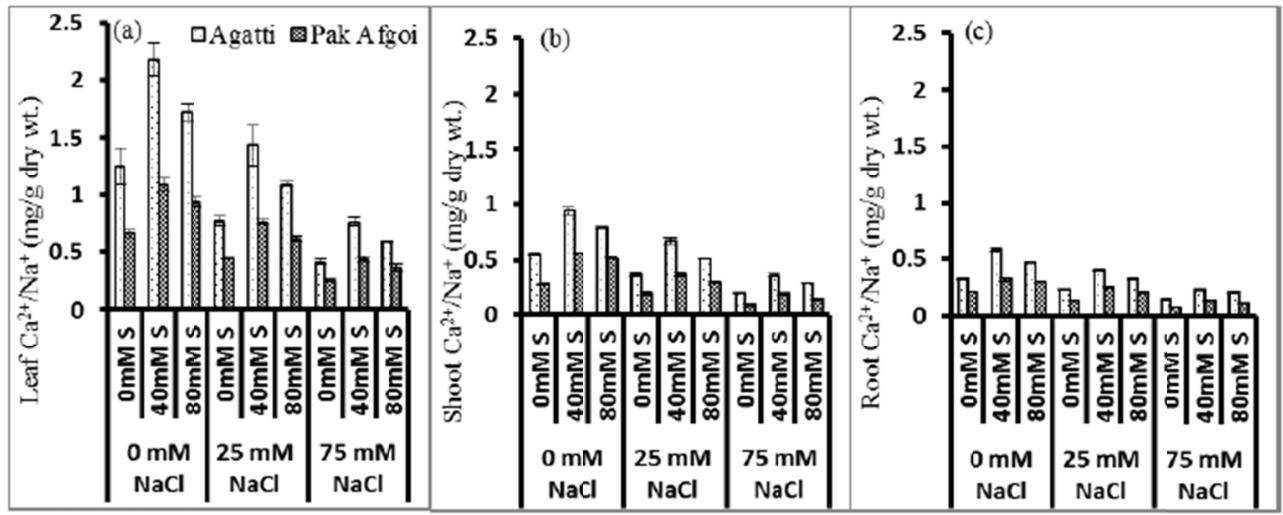

Figure 10. Effect of different levels of sulfur (S) on $\mathrm{Ca}^{2+} / \mathrm{Na}^{+}$content in leaf (a) shoot (b) root (c) of different maize (Zea mays L.) cultivars under saline conditions

Table 6. Mean squares from analysis of variance (ANOVA) of the data for $\mathrm{Ca}^{2+} / \mathrm{Na}^{+}$ratio and nitrate $\left(\mathrm{NO}_{3}^{-}\right)$of maize subjected to different levels of salinity and sulfur

\begin{tabular}{|c|c|c|c|c|c|c|c|}
\hline SOV & df & Leaf $\mathrm{Ca}^{2+} / \mathrm{Na}^{+}$ratio & Shoot $\mathrm{Ca}^{2+} / \mathrm{Na}^{+}$ratio & Root $\mathrm{Ca}^{2+} / \mathrm{Na}^{+}$ratio & Leaf $\mathrm{NO}_{3}^{-}$ & Shoot $\mathrm{NO}_{3}^{-}$ & Root $\mathrm{NO}_{3}^{-}$ \\
\hline Variety (V) & 1 & $3.60 * * *$ & $0.68 * * *$ & $0.24 * * *$ & $0.06 * * *$ & $0.057 * * *$ & $0.05 * * *$ \\
\hline Salinity (Sa) & 2 & $3.14 * * *$ & $0.68 * * *$ & $0.22 * * *$ & $0.07 * * *$ & $0.029 * * *$ & $0.05 * * *$ \\
\hline Sulfur (S) & 2 & $1.02 * * *$ & $0.25 * * *$ & $0.084 * * *$ & $0.05 * * *$ & $0.027 * * *$ & $0.045 * * *$ \\
\hline $\mathrm{V} \times \mathrm{Sa}$ & 2 & $0.39 * * *$ & $0.03 * * *$ & $0.01 * * *$ & $1.32 \mathrm{e}-4 \mathrm{~ns}$ & $3.88 \mathrm{e}-4 \mathrm{~ns}$ & $0.0019 * * *$ \\
\hline $\mathrm{V} \times \mathrm{S}$ & 2 & $0.13 * *$ & $0.012 * * *$ & $0.0055 * * *$ & $1.32 \mathrm{e}-4 \mathrm{~ns}$ & $0.0012 *$ & $2.06 \mathrm{e}-4 \mathrm{~ns}$ \\
\hline $\mathrm{Sa} \times \mathrm{S}$ & 4 & $0.064 * *$ & $0.017 * * *$ & $0.0046 * * *$ & $0.0045 * * *$ & $0.0025 * * *$ & $0.0068 * * *$ \\
\hline $\mathrm{V} \times \mathrm{Sa} \times \mathrm{S}$ & 4 & $0.011 \mathrm{~ns}$ & $9.16 \mathrm{e}-4 \mathrm{~ns}$ & $0.0011 * *$ & $1.32 \mathrm{e}-4 \mathrm{~ns}$ & $0.0014 * *$ & $2.28 \mathrm{e}-4 \mathrm{~ns}$ \\
\hline Error & 36 & 0.016 & $6.87 \mathrm{E}-04$ & 2.64E-04 & $3.48 \mathrm{E}-04$ & $3.05 \mathrm{E}-04$ & $1.71 \mathrm{E}-04$ \\
\hline
\end{tabular}

Note. ${ }^{*}, * *, * * *=$ significant at $0.05,0.01$ and 0.001 levels, respectively. $\mathrm{ns}=$ non-significant.

Abbreviation: Exponent (e), $\mathrm{Ca}^{2+}=$ Calcium, $\mathrm{Na}^{+}=$Sodium, $\mathrm{NO}_{3}{ }^{-}=$Nitrate.

\subsubsection{Nitrate $\left(\mathrm{NO}_{3}^{-}\right)$}

The application of salinity reduced the nitrate $\left(\mathrm{NO}_{3}{ }^{-}\right)$contents in all studied maize organs (leaf, shoot and root). The maximum reduction in nitrate contents was found at $75 \mathrm{mM}$ salt level (Figure 11).

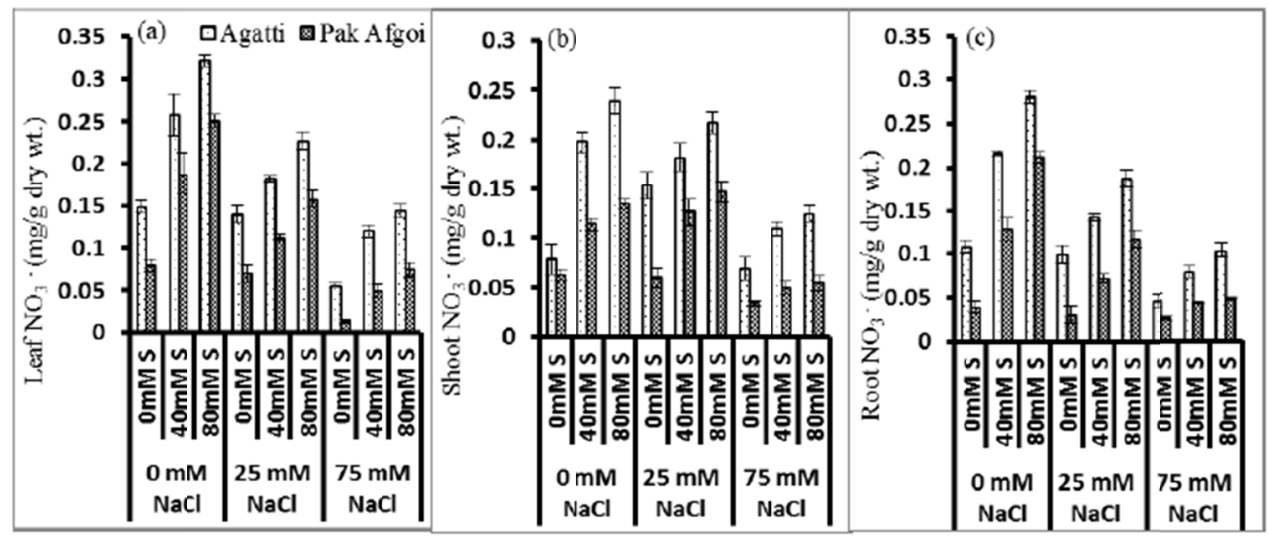

Figure 11. Effect of different levels of sulfur (S) on $\mathrm{NO}_{3}{ }^{-}$content in leaf (a) shoot (b) root (c) of different maize (Zea mays L.) cultivars under saline conditions

However, both varieties responded differently to salt application. In root, a statistically significant $\mathrm{V} \times \mathrm{Sa}$ interaction was found while in shoot and leaf, $\mathrm{V} \times \mathrm{Sa}$ interaction was non-significant (Table 6). The application of 
sulfur $(40,80 \mathrm{mM})$ improved the nitrate $\left(\mathrm{NO}_{3}{ }^{-}\right)$contents at all studied salt levels. It was evident from statistically significant $\mathrm{Sa} \times \mathrm{S}$ interactive effect for leaf, root and shoot (Table 6). Moreover, sulfur at $40 \mathrm{mM}$ level improved the salt tolerance in maize plants in both varieties (Agaitti, 2003; Pak Afgoi, 2003). A statistically significant V $\times$ $\mathrm{Sa} \times \mathrm{S}$ interaction was found in shoot while in leaf and root this interaction was non-significant (Table 6).

\subsubsection{Phosphate $\left(\mathrm{PO}_{4}{ }^{3-}\right)$}

A marked reduction in phosphate $\left(\mathrm{PO}_{4}{ }^{3-}\right)$ contents was found by salt application (Figure 12). However, sulfur application improved the phosphate $\left(\mathrm{PO}_{4}{ }^{3-}\right)$ contents in salt tolerant (Agaitti, 2003) and salt sensitive (Pak Afgoi, 2003) maize cultivars. A statistically significant $\mathrm{V} \times \mathrm{S}$ interaction for maize leaf revealed this fact, while in shoot and $\operatorname{root} \mathrm{V} \times \mathrm{S}$ interaction was found non-significant (Table 7).

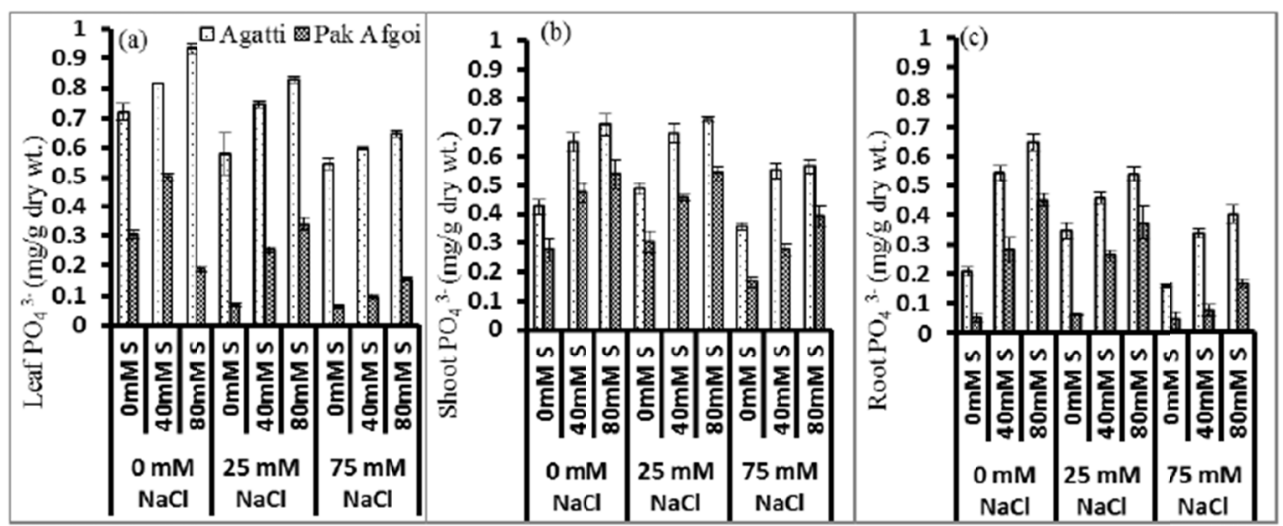

Figure 12. Effect of different levels of sulfur (S) on $\mathrm{PO}_{4}{ }^{3-}$ content in leaf (a) shoot (b) root (c) of different maize (Zea mays L.) cultivars under saline conditions

Moreover, sulfur application induced the salt tolerance in maize cultivars. It was shown by statistically significant $\mathrm{Sa} \times \mathrm{S}$ interaction for leaf and root at all studied salt levels $(25,75 \mathrm{mM})$ while in shoot this interaction was found non-significant (Table 7). In both varieties the application of sulfur improved the phosphate $\left(\mathrm{PO}_{4}{ }^{3-}\right)$ contents under salt stress conditions. It was shown by statistically significant $\mathrm{V} \times \mathrm{Sa} \times \mathrm{S}$ interaction for leaf and root while for shoot, this interaction was non-significant (Table 7). These findings revealed that sulfur application at $80 \mathrm{mM}$ improved the salt tolerance by improving the phosphate $\left(\mathrm{PO}_{4}{ }^{3-}\right)$ contents in both maize cultivars (Figure 12).

Table 7. Mean squares from analysis of variance (ANOVA) of the data for phosphate $\left(\mathrm{PO}_{4}{ }^{3-}\right)$ and sulfate $\left(\mathrm{SO}_{4}{ }^{2-}\right)$ of maize subjected to different levels of salinity and sulfur

\begin{tabular}{|c|c|c|c|c|c|c|c|}
\hline SOV & $\mathrm{df}$ & Leaf $\mathrm{PO}_{4}{ }^{3-}$ & Shoot $\mathrm{PO}_{4}{ }^{3-}$ & Root $\mathrm{PO}_{4}{ }^{3-}$ & Leaf $\mathrm{SO}_{4}{ }^{2-}$ & Shoot $\mathrm{SO}_{4}{ }^{2-}$ & Root $\mathrm{SO}_{4}{ }^{2-}$ \\
\hline Variety (V) & 1 & $3.29 * * *$ & $0.50 * * *$ & $0.58 * * *$ & $1340.01 * * *$ & $2506.35 * * *$ & $663.89 * * *$ \\
\hline Salinity $(\mathrm{Sa})$ & 2 & $0.23 * * *$ & $0.12 * * *$ & $0.14 * * *$ & $757.22 * * *$ & $327.25 * * *$ & $411.105 * * *$ \\
\hline Sulfur (S) & 2 & $0.099 * * *$ & $0.28 * * *$ & $0.37 * * *$ & $1813.53 * * *$ & $661.81 * * *$ & $950.16 * * *$ \\
\hline $\mathrm{V} \times \mathrm{Sa}$ & 2 & $3.19 \mathrm{e}-5 \mathrm{~ns}$ & $0.0030 \mathrm{~ns}$ & $1.27 \mathrm{e}-4 \mathrm{~ns}$ & $0.018 \mathrm{~ns}$ & $18.38 *$ & $29.06 * *$ \\
\hline $\mathrm{V} \times \mathrm{S}$ & 2 & $0.02 * * *$ & $0.0036 \mathrm{~ns}$ & $0.0036 \mathrm{~ns}$ & $0.018 \mathrm{~ns}$ & $14.76 \mathrm{~ns}$ & $80.13 * * *$ \\
\hline $\mathrm{Sa} \times \mathrm{S}$ & 4 & $0.026 * * *$ & $0.0016 \mathrm{~ns}$ & $0.024 * * *$ & $26.63 *$ & $8.79 \mathrm{~ns}$ & $48.14 * * *$ \\
\hline $\mathrm{V} \times \mathrm{Sa} \times \mathrm{S}$ & 4 & $0.026 * * *$ & $8.70 \mathrm{e}-4 \mathrm{~ns}$ & $0.0077 * *$ & $0.018 \mathrm{~ns}$ & $12.07 \mathrm{~ns}$ & $3.28 \mathrm{~ns}$ \\
\hline Error & 36 & 0.0013 & 0.0026 & 0.0018 & 7.35 & 4.85 & 4.99 \\
\hline
\end{tabular}

Note. $* * *, * * *=$ significant at $0.05,0.01$ and 0.001 levels, respectively. $\mathrm{ns}=$ non-significant.

Abbreviation: Exponent (e), $\mathrm{PO}_{4}{ }^{3-}=$ Phosphate, $\mathrm{SO}_{4}{ }^{2-}=$ Sulfate.

\subsubsection{Sulfate $\left(\mathrm{SO}_{4}{ }^{2-}\right)$}

Results have shown that sulfate $\left(\mathrm{SO}_{4}{ }^{2-}\right)$ contents were decreased by high levels of salinity $(75 \mathrm{mM})$ in both studied maize varieties. It was evident from statistically significant $\mathrm{V} \times \mathrm{Sa}$ interaction for shoot and root, and 
non-significant for leaf (Table 7). The application of sulfur improved the sulfate $\left(\mathrm{SO}_{4}{ }^{2-}\right)$ contents in both maize varieties, while the maximum improvement in sulfate $\left(\mathrm{SO}_{4}{ }^{2-}\right)$ contents was found at $80 \mathrm{M}$ sulfur. It was shown by statistically significant $\mathrm{Sa} \times \mathrm{S}$ interaction for leaf and root (Table 7). Although, sulfur improved the sulfate $\left(\mathrm{SO}_{4}{ }^{2-}\right)$ contents in salt tolerant variety (Agaitti, 2003), however, salt sensitive variety (Pak Afgoi, 2003) also improved the sulfate $\left(\mathrm{SO}_{4}{ }^{2-}\right)$ contents at all studied salt levels $(25,75 \mathrm{mM})$. In leaf sulfate $\left(\mathrm{SO}_{4}{ }^{2-}\right)$ contents were found vary high as compared to shoot and root (Figure 13).

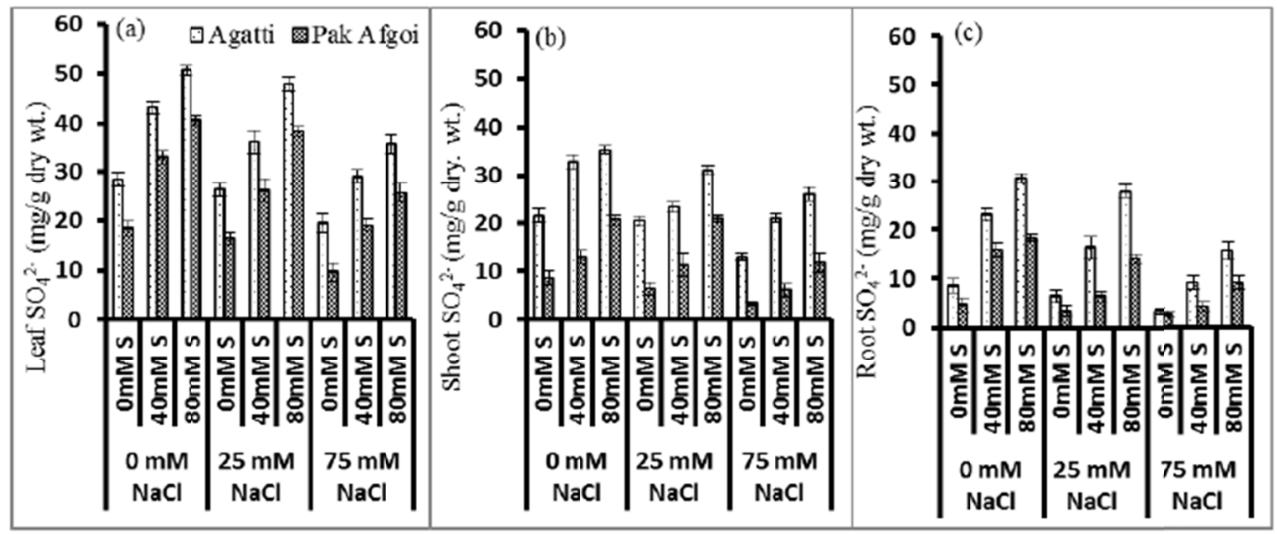

Figure 13. Effect of different levels of sulfur (S) on $\mathrm{SO}_{4}{ }^{2-}$ content in leaf (a) shoot (b) root (c) of different maize (Zea mays L.) cultivars under saline conditions

\section{Discussion}

Osmoprotectants (also called compatible osmolytes) are the organic molecules of small size, have neutral charges and less toxic at elevated concentration (Lang, 2007). Osmolytes not only regulate osmosis but also balance the cell volume by linking to the cytoplasmic organelles, without any disturbance to the usual plant metabolism and fold the proteins to endure the harsh effects of environmental (biotic or abiotic) stresses (Verbruggen \& Hermans, 2008). These osmolytes also stabilize the membrane proteins, prevent dehydration, nutrient homeostasis and regulate the osmotic potential inside the plant cell (Burg \& Ferraris, 2008).

The current study showed that glycine betaine contents were high in salt stress condition. The findings of this study is supported by previous investigation that confer the accumulation of glycine betaine develops salt tolerance in plants (Sakamoto \& Murata, 2002). Glycine betaine accumulation protects the plants from toxic effects of salinity by preventing oxidative stress (Chen \& Murata, 2008). Moreover, it plays significant roles in osmotic adjustment, stabilization of embedded proteins, protection of chloroplast and PS II complex and in reducing the reactive oxygen species produced under oxidative stress conditions (Cha-Um \& Kirdmanee, 2010). The application of sulfur supported the glycine betaine accumulation in both studied maize cultivars. This may be due to the reason that enzymes containing sulfur promote the biosynthesis of glycine betaine (Rathinasabapathi et al., 1997). Hence, sulfur application develops salt tolerance in plants by increasing the glycine betaine contents in plants.

The results of this study showed that proline concentration was higher in salt tolerant maize cultivar (Agaitti, 2003) in comparison to the salt sensitive maize variety. This finding is supported by previous researches on various crops i.e. rice, alfalfa, maize, pigeon pea and potato (Rahnama \& Ebrahimzadeh, 2004; Waheed et al., 2006; Shereen et al., 2007; Cha-um \& Kirdmanee, 2010). Proline is very much helpful in enduring the adverse conditions of environmental stresses. Proline serves as cytoplasmic osmoticum. Under salt stress condition, high accumulation of proline has been reported in previous studies (Miller et al., 2010). Moreover, it serves as nitrogen reservoir in the periods of restricted growth, hydrate the polymers and scavenge the reactive oxygen species (Kavi Kishor et al., 1995). However, in this study, sulfur application lowered the proline contents in both studied maize varieties (Agaitti, 2003; Pak Afgoi, 2003). This may be due to the reason that excessive amount of proline creates toxic effects in the plants (Jain et al., 2000). Hence, sulfur metabolites regulate the osmolyte concentration for developing salt tolerance in the plants.

The present study showed that salinity increased the total soluble sugars contents to induce salt tolerance in crop plants. It was due to the reason that accumulation of soluble sugars reduces the osmotic potential, water potential, turgidity in plant cell and osmotic adjustment by increasing the storage reserves for the normal functions of 
plants under stress conditions (Siringam et al., 2012). Moreover, soluble sugars serve as chelating agent that bound $\mathrm{Na}^{+}$with starches and lower the toxic effects of salt on the plants (Xiao et al., 2009). The application of sulfur also increased the soluble sugars contents in both maize cultivars at all levels of treatments. It was supported by the findings of Lunde et al. (2008) who reported the reduction in soluble sugar contents by sulfur deficiency.

In this study, it was found that salinity increased the total soluble protein contents in both studied maize cultivars. It was supported by earlier researches (Chen et al., 2007; Kapoor \& Srivastava, 2010). Soluble proteins help to raise the nitrogen level in plants that promotes growth and development under stress conditions. In addition, soluble proteins perform a significant role in osmotic adjustment (Ashraf \& Harris, 2004). Sibole et al. (2003) found that by application of salinity $(10,50,100,200 \mathrm{mM})$, the soluble protein contents were increased in the clover plant (Medicago citrna L.). The accumulation of soluble protein contents by salt application has been reported in various plants i.e. barley, maize, sunflower, rice and mung bean (Khosravinejad et al., 2009; Kapoor \& Srivastava, 2010). This study showed that the application of sulfur improved the soluble protein contents in maize plants. It may be due to the reason that sulfur is an important part of amino acids the building blocks of proteins (Gardner et al., 1985). Different metabolites of sulfur (i.e. cysteine, thiol) protect the structure of proteins. Hence, sulfur helps in forming the structure and function of proteins in the stress conditions (Malhi \& Leach, 2000).

It was found that salt stress enhanced the total free amino acid contents in maize plants. In stress conditions, total free amino acid contents become very high that protects the proteins from degradation (Mansour, 2000). Moreover, this study showed that salt tolerant maize cultivar accumulated high level of total free amino acid in comparison to salt sensitive maize variety. These findings have been supported by previous studies (Ashraf and Tufail, 1995; Ashraf \& Fatima, 2004). The application of sulfur improved various amino acid contents in maize plants as sulfur is the constituent of many important amino acids forming various structural and functional proteins in plants (Giovaneli, 1987).

Salt stress causes the disturbance in availability, absorption and transport of nutritional contents in plants (Munns \& Tester, 2008). In this study, salinity reduced the beneficial nutrients $\left(\mathrm{K}^{+}, \mathrm{Ca}^{2+}, \mathrm{NO}_{3}{ }^{-}, \mathrm{PO}_{4}{ }^{3-}, \mathrm{SO}_{4}{ }^{2-}, \mathrm{K}^{+} / \mathrm{Na}^{+}\right.$, $\left.\mathrm{Ca}^{2+} / \mathrm{Na}^{+}\right)$in maize plants. It may be due to the reason that salt stress causes the disturbance in external osmotic potential that imbalance the nutrient contents in plants (Murillo-Amador et al., 2002). The imbalance in nutrient contents has been reported in various crops e.g. Lycopersicon esculentum, Spinacia oleracea, Physalis peruviana, as well as in Zea mays (Miranda et al., 2010; Collado et al., 2010).

This study revealed that salt stress increased the sodium $\left(\mathrm{Na}^{+}\right)$contents in the maize plants which are in accordance to the findings of Fortmeier et al. (1995). The rise in sodium $\left(\mathrm{Na}^{+}\right)$contents decreased the plant growth in both studied maize cultivars (Agaitti, 2003; Pak Afgoi, 2003). It may be due to the reason that high sodium $\left(\mathrm{Na}^{+}\right)$contents forms ion-pair and precipitates other ions in plant cell (Hu et al., 2005). The reduction in $\mathrm{Ca}^{2+}, \mathrm{K}^{+}, \mathrm{K}^{+} / \mathrm{Na}^{+}$and $\mathrm{Ca}^{2+} / \mathrm{Na}^{+}$has been reported in this study. The elevated concentration of sodium $\left(\mathrm{Na}^{+}\right)$ changes the root permeability and reduces the uptake of calcium $\left(\mathrm{Ca}^{2+}\right)$ in plants (Greenway \& Munns, 1980). This may be due to the competition in uptake of sodium $\left(\mathrm{Na}^{+}\right)$and calcium $\left(\mathrm{Ca}^{2+}\right)$ contents and due to reduction in soil water potential affecting root pressure (Sonnevelt et al., 1975). Moreover, high concentration of sodium $\left(\mathrm{Na}^{+}\right)$negatively uptake the potassium $\left(\mathrm{K}^{+}\right)$resulting in reduction in carbon fixation, photosynthetic apparatus and ultimately reduces the photosynthesis in plants (Akram et al., 2010). The results of this study revealed that salt tolerant cultivar (Agaitti, 2003) accumulated low sodium $\left(\mathrm{Na}^{+}\right)$and high potassium $\left(\mathrm{K}^{+}\right)$and calcium $\left(\mathrm{Ca}^{2+}\right)$ contents in comparison to salt sensitive maize variety (Pak Afgoi, 2003). Therefore, Agaitti (2003) showed high $\mathrm{K}^{+} / \mathrm{Na}^{+}$and $\mathrm{Ca}^{2+} / \mathrm{Na}^{+}$ratio. This may be due to the reason that salt tolerant variety compartmentalizes the sodium $\left(\mathrm{Na}^{+}\right)$in the plants thus transport the potassium and calcium (Munns et al., 2006). Thus, salt tolerant cultivar has high $\mathrm{K}^{+} / \mathrm{Na}^{+}$ratio. It was supported by previous studies (Song et al., 2009). In salt tolerant variety the restricted uptake of $\mathrm{Na}^{+}$ions maintains plant homeostasis and ultimately overall plant growth. While in salt sensitive variety, plant growth reduced due to disturbance in nutrient homeostasis. These findings are in accordance to previous researches (Eker et al., 2006; Riffat \& Ahmad, 2018). Results showed that application of sulfur lowered the $\mathrm{Na}^{+}$ions and improved the $\mathrm{Ca}^{2+}, \mathrm{K}^{+}, \mathrm{K}^{+} / \mathrm{Na}^{+}$and $\mathrm{Ca}^{2+} / \mathrm{Na}^{+}$in the maize plants. Sulfur helps in maintaining nutrient homeostasis in plants and induces salt tolerance (Singh et al., 2011). Sulfur application increases the $\mathrm{Ca}^{2+}$ and $\mathrm{K}^{+}$ions and decreases the harmful effects of $\mathrm{Na}^{+}$ions in the plants. This results in high $\mathrm{K}^{+} / \mathrm{Na}^{+}$and $\mathrm{Ca}^{2+} / \mathrm{Na}^{+}$ratio that indicate salt tolerance. Thus application of sulfur improves the crop quality and growth and development by maintaining proper nutrient homeostasis in plants under stressful environment (Badr et al., 2002; Prasad et al., 2003). 
Results showed that salinity reduced nitrate $\left(\mathrm{NO}_{3}{ }^{-}\right)$contents in maize plants. It was supported by previous findings of Samra (1985). It may be due to the reason that $\mathrm{Na}^{+}$ions cause slow assimilation of nitrate $\left(\mathrm{NO}_{3}{ }^{-}\right)$ contents. Moreover, salt stress shifts the reduction of nitrate from leaf to root (Frechill et al., 2001; Ullrich, 2002), that disturbs the proper availability of nitrate $\left(\mathrm{NO}_{3}{ }^{-}\right)$to the other parts of plants. The application of sulfur improved the nitrate $\left(\mathrm{NO}_{3}{ }^{-}\right)$contents in both studied maize varieties. It was in accordance to the previous studies. Reuveny et al. (1980) reported that the deficiency of sulfur causes the reduction in nitrate reductase activity. However, sulfur application improves the nitrogen metabolism and ultimately improves the nitrate contents in stress conditions (Sexton et al., 1993).

Salt stress also reduced the phosphate $\left(\mathrm{PO}_{4}{ }^{3-}\right)$ contents in maize plants. Champagnol (1979) reported that salt stress reduced the phosphate $\left(\mathrm{PO}_{4}{ }^{3-}\right)$ nutrition in the plants. However, sulfur application at low concentration improved the phosphorous contents in maize plants. These findings are supported by previous researches on various crops i-e. wheat, chickpea and maize, (Islam et al., 2011; Riffat, 2017; Riffat \& Ahmad, 2018). Results revealed that salt stress reduced the sulfate $\left(\mathrm{SO}_{4}{ }^{2-}\right)$ contents in both studied maize varieties. Riffat \& Ahmad (2018) reported that high concentration of salts reduced the sulfate $\left(\mathrm{SO}_{4}{ }^{2-}\right)$ contents. While, the sulfur application improved the sulfate $\left(\mathrm{SO}_{4}{ }^{2-}\right)$ contents in the maize plants.

\section{Conclusions and Recommendations}

Salt stress caused changes in the organic and inorganic osmolytes in the plants. The imbalance in nutrient contents disturbs the normal plant metabolism. To overcome the adverse effects of salinity some natural osmoprotectants get accumulated in the maize plants. Among these organic osmolytes, glycine betaine, proline, total soluble sugars, total soluble proteins and total free amino acids has considerable importance. The application of sulfur $(40 \mathrm{mM})$ not only balanced the organic osmolytes contents by lowering the higher accumulation of proline to avoid toxic effects but also induced salt tolerance in maize plants. Among the inorganic osmolytes, salt stress increased the $\mathrm{Na}^{+}$contents and lowered the beneficial osmolytes in the maize plants. However, sulfur application at $40 \mathrm{mM}$ proved very effective in improving beneficial osmolytes $\left(\mathrm{K}^{+}, \mathrm{Ca}^{2+}\right.$, $\mathrm{NO}_{3}{ }^{-}, \mathrm{PO}_{4}{ }^{3-}, \mathrm{SO}_{4}{ }^{2-}, \mathrm{K}^{+} / \mathrm{Na}^{+}$and $\left.\mathrm{Ca}^{2+} / \mathrm{Na}^{+}\right)$in the plants. Hence, it is recommended that sulfur at $40 \mathrm{mM}$ is very much effective in balancing organic and inorganic osmolytes for improving salt tolerance potential.

\section{References}

Akram, M., Ashraf, M. Y., Ahmad, R., Waraich, E. A., Iqbal, J., \& Mohsan, M. (2010). Screening for salt tolerance in maize (Zea mays L.) hybrids at an early seedling stage. Pakistan Journal of Botany, 42,141-154.

Ashraf, M., \& Fatima, H. (1995). Responses of salt tolerant and salt sensitive lines of safflower (Carthamus tinctorius L.). Acta Physiologia Plantarum., 17, 61-71.

Ashraf, M., \& Harris, P. J. C. (2004). Potential biochemical indicators of salinity tolerance in plants. Plant Sciences, 166, 3-16. https://doi.org/10.1016/j.plantsci.2003.10.024

Ashraf, M., \& Tufail, M. (1995). Variation in salinity tolerance in sunflower (Helianthus annuus L.). Journal of Agronomy and Soil Sciences, 174, 351-62. https://doi.org/10.1111/j.1439-037X.1995.tb01122.x

Badr, Z., Ali, A., Salim, M., \& Niazi, B. H. (2002). Role of sulfur for potassium/sodium ratio in sunflower under saline conditions. Helia, 25, 69-78. https://doi.org/10.2298/HEL0237069B

Bates, L. S., Waldren R. P., \& Teare, I. D. (1973). Rapid determination of free proline for water stress studies. Plant Soil, 39, 205-207. https://doi.org/10.1007/BF00018060

Bukhsh, M. A. A. H. A., Ahmad, R., Iqbal, J., Rehman, A., Hussain, S., \& Ishaque, M. (2011). Potassium application reduces bareness in different maize hybrids under crowding stress conditions. Pakistan Journal Agricultural Sciences, 48, 31-37.

Burg, M. B., \& Ferraris, J. D. (2008). Intracellular organic osmolytes: Function and regulation. Journal of Biological Chemistry, 283, 7309-7313.https://doi.org/10.1074/jbc.R700042200

Champagnol, F. (1979). Relationships between phosphate nutrition of plants and salts toxicity. Phosphorus Agriculture, 76, 35-43.

Cha-Um, S., \& Kirdmanee, C. (2010). Effect of glycinebetaine on proline, water use, and photosynthetic efficiencies, and growth of rice seedlings under salt stress. Turkish Journal of Agriculture and Forestry, 34, 517-527. https://doi.org/10.3906/tar-0906-34 
Chen, C., Tao, C., Peng, H., \& Ding, Y. (2007). Genetic analysis of salt stress responses in asparagus bean (Vigna unguiculata L. ssp. Sesquipedalis verdc.). Journal of Heredity, 98, 655-665. https://doi.org/10.1093/jhered/ esm084

Chen, T. H. H., \& Murata, N. (2008). Glycinebetaine: an effective protectant against abiotic stress in plants. Trends in Plant Sciene, 13, 499-505. https://doi.org/10.1016/j.tplants.2008.06.007

Collado, M. B., Arturi, M. J., Aulicino, M. B., \& Molina, M. C. (2010). Identification of salt tolerance in seedling of maize (Zea mays L.) with the cell membrane stability trait. International Research Journal of Plant Sciences, 1, 126-132.

Eker, S., Comertpay, K. O., Ulger, A. C., Ozturk, L., \& Cakmak, I. (2006). Effect of salinity stress on dry matter production and ion accumulation in hybrids maize varieties. Turkish Journal of Agriculture and Forestry, 30, 365-373.

Farooq, M., Hussain, M., Wakeel, \& Siddique, K. H. M. (2015). Salt stress in maize: Effects, resistance mechanisms, and management: A review. Agronomy for Sustainable Development, 35, 461. https://doi.org/ 10.1007/s13593-015-0287-0

Fortmeier, R., \& Schubert, S. (1995). Salt tolerance of maize (Zea mays L.): The role of Sodium exclusion. Plant Cell Environment, 18, 1041-1047. https://doi.org/10.1111/j.1365-3040.1995.tb00615.x

Frechill, S., Lasa, B., Ibarretxe, L., Lamsfus, C., \& Aparicio, P. T. (2001). Pea responses to saline stress is affected by the source of nitrogen nutrition (ammonium or nitrate). Plant Growth Regulation, 35, 171-179. https://doi.org/10.1023/A:1014487908495

Gardner, F. P., Pearce, R. B., \& Mitchel, R. L. (1985). Growth and Development. In: Physiology of Crop Plants. Iowa State University Press, Ames, Iowa.

Giovaneli, J. (1987). Sulfur amino acids of plants: An overview. Methods in Enzymology, 143, 419-426. https://doi.org/10.1016/0076-6879(87)43073-5

Greenway, H., \& Munns, A. (1980). Mechanism of salt tolerance in non-halophytes. Annual Review of Plant Physiology, 31, 149-190. https://doi.org/10.1146/annurev.pp.31.060180.001053

Grieve, C. M., \& Grattan, S. R. (1983). Rapid assay for determination of water soluble quaternary ammonium compounds. Plant Soil, 70, 303-307. https://doi.org/10.1007/BF02374789

$\mathrm{Hu}, \mathrm{Y} .$, \& Schmidhalter, U. (2005). Drought and salinity: A comparison of their effects on mineral nutrition of Plants. Journal of Plant Nutrition and Soil Sciences, 168, 541-549. https://doi.org/10.1002/jpln.200420516

Iqbal, N., Umer, S., \& Nazar, R. (2014). Maipulating osmolytes for breeding salinity tolerant plants. Emerging technologies and management of crop stress tolerance, 2, 385-404. https://doi.org/10.1016/B978-0-12800875-1.00016-8

Islam, M., Mohsan, S., Afzal, S., Ali, S., Akmal, M., \& Khalid, R. (2011). Phosphorus and sulfur application improves the chickpea productivity under rainfed conditions. International Journal of Agriculture and Biology, 13, 713-718.

Jain, M. J., Mathur, G. M., Koul, S. K., \& Sarin, N. S. (2000). Ameliorative effects of proline on salt stress-induced lipid peroxidation in cell lines of groundnut (Arachis hypogaea L.) Plant Cell Reports, 20, 463-8. https://doi.org/10.1007/s002990100353

Kapoor, K., \& Srivastava, A. (2010). Assessment of salinity tolerance of Vinga mungo var. Pu-19 using ex vitro and in vitro methods. Asian Journal of Biotechnology, 2, 73-85. https://doi.org/10.3923/ajbkr.2010.73.85

Kavi Kishor, P. B., Hong, Z. L., \& Miao, G. H. (1995). Over expression of 1-pyrroline5-carboxylate synthetase increases proline production and confers osmotolerance in transgenic plants. Plant Physiology, 108, 1378-1394. https://doi.org/10.1104/pp.108.4.1387

Khosravinejad, F., Heydari, R., \& Farboodnia, T. (2009). Effect of salinity on organic solutes contents in barley. Pakistan Journal of Biological Sciences, 12, 158-162. https://doi.org/10.3923/pjbs.2009.158.162

Kowalenko, C. G., \& Lowe, L. E. (1973). Determination of nitrates in soil extracts. Soil Science Society of America Proceedings, 37, 660. https://doi.org/10.2136/sssaj1973.03615995003700040051x

Lang, F. (2007). Mechanisms and significance of cell volume regulation. Journal of American College of Nutrition, 26, 613-623. https://doi.org/10.1080/07315724.2007.10719667 
Lunde, C., Zygadlo, A., Simonsen, H. T., Nielsen, P. L., Blennow, A., \& Haldrup, A. (2008). Sulfur starvation in rice: the effect on photosynthesis, carbohydrate metabolism, and oxidative stress protective pathways. Physiologia Plantarum, 134, 508-21.https://doi.org/10.1111/j.1399-3054.2008.01159.x

Machado, R. M. A., \& Serralheiro, R. P. (2017). Review soil salinity: effect on vegetable crop growth management practices to prevent and mitigate soil salinization. Horticulturae, 3, 30. https://doi.org/ 10.3390/horticulturae 3020030

Malhi, H., \& Leach, D. (2000). Restore Canola Yields by Correcting Sulfur Deficiency in the Growing Season. Proc. $12^{\text {th }}$ Annual Meeting and Conference. Sustainable Farming in the New Millennium. Saskatchewan Soil Conservation Association, Regina, SK, Canada.

Mansour, M. M. F. (2000). Nitrogen containing compounds and adaptation of plants to salinity stress. Biologia Plantarum, 43, 491-500. https://doi.org/10.1023/A:1002873531707

Miller, G., Suzuki, N., Ciftci-Yilmaz, S., \& Mittler, R. (2010). Reactive oxygen species homeostasis and signaling during drought and salinity stresses. Plant Cell Environment, 33, 453-467. https://doi.org/10.1111/ j.1365-3040.2009.02041.x

Miranda, D., Fischer, G., \& Ulrichs, C. (2010). Growth of cape gooseberry (Physalis peruviana L.) plants affected by salinity. Journal of Applied Botany and Food Quality, 83, 175-181.

Munns, R., \& Tester, M. (2008). Mechanisms of salinity tolerance. Annual Review of Plant Biology, 59, 651-681. https://doi.org/10.1146/annurev.arplant.59.032607.092911

Munns, R., James, R. A., \& Lauchli, A. (2006). Approaches to increasing the salt tolerance of wheat and other cereals. Journal of Experimental Botany, 57, 1025-1043. https://doi.org/10.1093/jxb/erj100

Murillo-Amador, B., Lopez-Aguilar, R., Kaya, C., Larrinaga-Mayoral, J., \& Flores-Hernandez, A. (2002). Comparative effects of $\mathrm{NaCl}$ and polyethylene glycol on germination, emergence and seedling growth of cowpea. Journal of Agronomy and Crop Sciences, 188, 235-247. https://doi.org/10.1046/j.1439-037X. 2002.00563.x

Nahar, K., Hasanuzzaman, M., \& Fujita, M. (2016). Roles of Osmolytes in Plant Adaptation to Drought and Salinity. In N. Iqbal, R. Nazar, \& A. N. Khan (Eds.), Osmolytes and Plants Acclimation to Changing Environment: Emerging Omics Technologies (pp. 37-68). Springer, New Delhi. https://doi.org/10.1007/ 978-81-322-2616-1_4

Nazar, R., Khan, M. I., Iqbal, N., Masood, A., \& Khan, N. A. (2014). Involvement of ethylene in reversal of salt-inhibited photosynthesis by sulfur in mustard. Physiologia Plantarum, 152, 331-344. https://doi.org/ 10.1111/ppl.12173

Parida, A. K., \& Das, A. B. (2005). Salt tolerance and salinity effects on plants: A review. Ecotoxicol Environmental Safety, 60, 324-49. https://doi.org/10.1016/j.ecoenv.2004.06.010

Pimentel, D., Berger, B., Filiberto, D., Newton, M., Wolfe, B., Karabinakis, E., ... Nandaopal, S. (2004). Water Resources: Agricultural and Environmental Issues. Bio Science, 54, 909-918. https://doi.org/10.1641/ 0006-3568(2004)054[0909:WRAAEI]2.0.CO;2

Prasad, B. (2003). Effect of direct and residual effects of different $\mathrm{S}$ fertilizers on groundnut and wheat cropping system on typic haplaquent soils. Journal of Plant Nutrition, 26, 997-1008. https://doi.org/10.1081/ PLN-120020071

Puniran-Hartley, N., Hartley, J., Shabala, L., \& Shabla, S. (2014). Salinity-induced accumulation of organic osmolytes in barley and wheat leaves correlates with increased oxidative stress tolerance: In planta evidence for cross-tolerance. Plant Phiological Biochemistry, 83, 32-9. https://doi.org/10.1016/j.plaphy.2014.07.005

Rahnama, H., \& Ebrahimzadeh, H. (2004). The effect of $\mathrm{NaCl}$ on proline accumulation in potato seedlings and calli. Acta Physiologia Plantarum, 26, 263-70. https://doi.org/10.1007/s11738-004-0016-9

Rathinasabapathi, B., Burnet, M., Russell, B., Gage, D. A., Liao, P. C., Nye, G. J., ... Hanson, A. D. (1997). Choline monooxygenase, an unusual iron-sulfur enzyme catalyzing the first step of glycine betaine synthesis in plants: Prosthetic group characterization and cDNA cloning. Proceedings of National Academy of Sciences USA, 94, 3454-3458. https://doi.org/10.1073/pnas.94.7.3454

Reuveny, Z., Dougall, D. K., \& Trinity, P. M. (1980). Regulatory coupling of nitrate and sulfate assimilation pathways in cultured tobacco cells. Proceedings of the National Academy of Sciences, 77, 6670-6672. 
Riffat, A. (2017). Effect of sulfur application on ionic contents and compatible osmolytes of maize (Zea mays L.) under saline conditions. $4^{\text {th }}$ ICONTES, Kuala Lumpur, August 5-6, 2017 . Retrieved from http://procedia.org/cpi/ICONTES-4-2110790

Riffat, A., \& Ahmad, M. S. A. (2018). Improvement in nutrient contents of maize (Zea mays L.) by sulfur modulation under salt stress. International Journal of Agronomy and Agricultural Research, 12, 100-117.

Riffat, A., \& Ahmed, M. S. A. (2016). Ameliorating adverse effects of salt stress on maize (Zea mays L.) cultivars by exogenous application of sulfur at seedling stage. Pakistan Journal of Botany, 48, 1323-3334.

Sakamoto, A., \& Murata, N. (2002). The role of glycine betaine in the protection of plants from stress: Clues from transgenic plants. Plant Cell Environment, 25, 163-71. https://doi.org/10.1046/j.0016-8025.2001. 00790.x

Samra, J. S. (1985). Sodicity tolerance of grapes with reference to the uptake of nutrients. Indian Journal of Horticulture, 43, 60-65.

Sexton, J., Bennett, J., Reynolds, S. B., \& Hocking, T. J. (1993). Effects of sulfur deficiency on nitrogen metabolism in sugar beet. Journal of Experimental Botany, 44(Supplement), 28.

Shereen, A., Ansari, R. U., Yamin, S., Raza, S., Mumtaz, S., \& Khan, M. A. (2007). Physiological responses of rice (Oryza sativa L.) to saline stress. Pakistan Journal of Botany, 39, 2527-534.

Sibole, J. V., Cabot, C., Poschenreder, C., \& Barcelo, J. (2003). Efficient leaf ion partitioning, an overriding condition for abscisic acid controlled stomatal and leaf growth responses to $\mathrm{NaCl}$ salinization in two legumes. Journal of Experimental Botany, 54, 2111-2119. https://doi.org/10.1093/jxb/erg231

Singh, J. S., Pandey, V. C., \& Singh, D. P. (2011). Efficient soil microorganisms: A new dimension for sustainable agriculture and environmental development. Agriculture Ecosystem and Environment, 140, 339-353. https://doi.org/10.1016/j.agee.2011.01.017

Siringam, K., Juntawong, N., Cha-um, S., Boriboonkaset, T., \& Kirdmanee, C. (2012). Salt tolerance enhance in indica rice (Oryza sativa L. spp. Indica) seedlings using exogenous sucrose supplementation. Plant Omics, $5,52-59$.

Song, J., Chen, M., Feng, G., Jia, Y., Wang, B., \& Zhang, F. (2009). Effect of salinity on growth, ion accumulation and the roles of ions in osmotic adjustment of two populations of Suaeda salsa. Plant Soil, 314, 133-141. https://doi.org/10.1007/s11104-008-9712-3

Sonnevelt, C., \& van den Ende, J. (1975). The effects of some salts on head weight and tipburn of lettuce and on fruit production and blossom end rot of tomatoes. Netherland Journal of Agricultural Sciences, 23, 192-201.

Spadaro, D., Yun, B. W., Spoel, S. H., Chu, C., Wang, Y. Q., \& Loake, G. J. (2010). The redox switch: Dynamic regulation of protein function by cysteine modifications. Physiologia Plantarum, 138, 360-371. https://doi.org/10.1111/j.1399-3054.2009.01307.x

Taiz, L., \& Zeiger, E. (2006). Plant Physiology (4th ed.). Sinauer Associates Inc. Sunderland, Massachusetts.

Tendon, H. L. S. (1993). Methods of Analysis of Soil, Plants, Water and Fertilizers. Fertilization Development and Consultation Organisation, New Delhi, India.

Ullrich, W. R. (2002). Salinity and Nitrogen Nutrition. In A. Läuchli, \& U. Lüttge (Eds.), Salinity: Environment-Plants-Molecules (pp. 229-248). Springer, Dordrecht. https://doi.org/10.1007/0-306-48155-3_11

Verbruggen, N., \& Hermans, C. (2008). Proline accumulation implants: A review. Amino Acids, 35, 753-759. https://doi.org/10.1007/s00726-008-0061-6

Waheed, A., Hafiz, I. A., Qadir, G., Murtaza, G., Mahmood, T., \& Ashraf, M. (2006). Effect of salinity on germination, growth, yield, ionic balance and solute composition of pigeon pea (Cajanus cajan (L.) Millsp.). Pakistan Journal of Botany, 38, 1103-17.

Wolf, B. (1982). A comprehensive system of leaf analysis and its use for diagnosing crop nutrient status. Communications in Soil Science and Plant Analysis, 13, 1035-1059. https://doi.org/10.1080/001036282093 67332

Xiao, B. Z., Chen, X., Xiang, C. B., Tang, N., Zhang, Q. F., \& Xiong, L. Z. (2009). Evaluation of seven function-known candidate genes for heir effects on improving drought resistance of transgenic rice under field conditions. Molecular Plant, 2, 73-83. https://oi.org/10.1093/mp/ssn068 
Yoshida, S., Forno, D. A., Cock, J. K., \& Gomez, K. A. (1976). Labortary Manual for Physiological Studies of Rice. IRRI, Los Banos.

\section{Copyrights}

Copyright for this article is retained by the author(s), with first publication rights granted to the journal.

This is an open-access article distributed under the terms and conditions of the Creative Commons Attribution license (http://creativecommons.org/licenses/by/4.0/). 